\title{
GPCA: A Probabilistic Framework for Gaussian Process Embedded Channel Attention
}

\author{
Jiyang Xie, Student Member, IEEE, Zhanyu Ma, Senior Member, IEEE, \\ Dongliang Chang, Guoqiang Zhang, Member, IEEE, and Jun Guo
}

\begin{abstract}
Channel attention mechanisms have been commonly applied in many visual tasks for effective performance improvement. It is able to reinforce the informative channels as well as to suppress the useless channels. Recently, different channel attention modules have been proposed and implemented in various ways. Generally speaking, they are mainly based on convolution and pooling operations. In this paper, we propose Gaussian process embedded channel attention (GPCA) module and further interpret the channel attention schemes in a probabilistic way. The GPCA module intends to model the correlations among the channels, which are assumed to be captured by beta distributed variables. As the beta distribution cannot be integrated into the end-to-end training of convolutional neural networks (CNNs) with a mathematically tractable solution, we utilize an approximation of the beta distribution to solve this problem. To specify, we adapt a Sigmoid-Gaussian approximation, in which the Gaussian distributed variables are transferred into the interval $[0,1]$. The Gaussian process is then utilized to model the correlations among different channels. In this case, a mathematically tractable solution is derived. The GPCA module can be efficiently implemented and integrated into the end-to-end training of the CNNs. Experimental results demonstrate the promising performance of the proposed GPCA module. Codes are available at https://github.com/PRIS-CV/GPCA
\end{abstract}

Index Terms-Deep learning, Bayesian learning, convolutional neural network, attention mechanism, Gaussian process

\section{INTRODUCTION}

$\mathrm{D}$ EEP neural networks have attracted great attention and improved the performance of various computer vision tasks from academic to industry including image classification [1], [2], [3], [4], object detection [5], semantic segmentation [6], |7], and image retrieval [8]. Convolutional neural networks (CNNs) as well-known deep neural network architectures are able to effectively capture the discriminative nonlinear patterns from images, extract feature representations, and generate multi-channel feature maps. These phenomena are mainly due to their deep and wide structures, and the introduction of the additional components including dropout [9] and attention schemes [10].

Attention mechanisms as the key components of CNN architectures are generally located behind specific convolutional layers, such as the last layers in each residual block of ResNet [2]. They have been utilized for feature recalibration with attention weights and widely applied to effectively improve the performance of the CNNs learned from large-scale datasets [11]. Previous work [11], [12], [13] have intensively studied the significance of the attention mechanisms. The attention scheme can not only inform the CNNs about their concentrations on an image or the extracted features, but also develop the feature representation of the image [13].

- J. Xie, Z. Ma, D. Chang, and J. Guo are with the Pattern Recognition and Intelligent System Lab., School of Artificial Intelligence, Beijing University of Posts and Telecommunications, China. E-mail: \{xiejiyang2013, mazhanyu, changdongliang, guojun\}@bupt.edu.cn

- G. Zhang is with the School of Electrical and Data Engineering, University of Technology Sydney, Australia. E-mail: guoqiang.zhang@uts.edu.au

(Corresponding author: Zhanyu Ma)

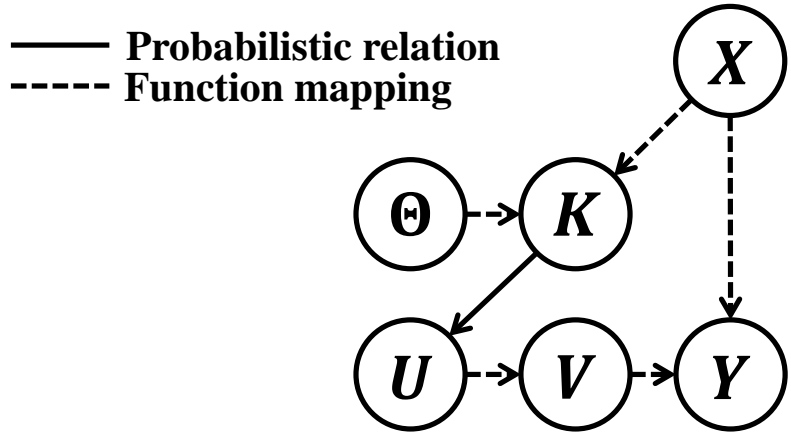

Fig. 1: Probabilistic graphical model of the Gaussian process embedded channel attention (GPCA) module. $\boldsymbol{X} \in$ $R^{C \times W \times H}$ are the input feature maps, where $C, W$, and $H$ are the number of channels, the width, and the height, respectively. Meanwhile, the final output $\boldsymbol{Y} \in R^{C \times W \times H}$ of the GPCA module is obtained by scaling $\boldsymbol{X}$ with the attention mask vector $\boldsymbol{V} \in R^{C}$. Each element in $\boldsymbol{V}$ is generated by the corresponding elements in $\boldsymbol{U} \in R^{C}$. Then, it is transferred and bounded into the interval $[0,1]$. $\boldsymbol{U}$ is the channel weight vector, optimized by a Gaussian process (GP) with the Gram matrix $\boldsymbol{K} \in R^{C \times C}$. $\boldsymbol{K}$ directly represents the channel correlations in $\boldsymbol{X}$ and is calculated by a kernel function with parameters $\Theta$.

Channel attention, which is a significant part of the attention mechanisms, aims to improve the quality of feature representations by precisely modeling the correlations between the channels [11]. It is able to enhance the informative and discriminative feature maps and suppress the useless and unhelpful ones simultaneously by learning the attention weights for each channel, which are optimized implicitly.

Relevant work [11], [13], [14], [15], [16], [17], [18], [19], 


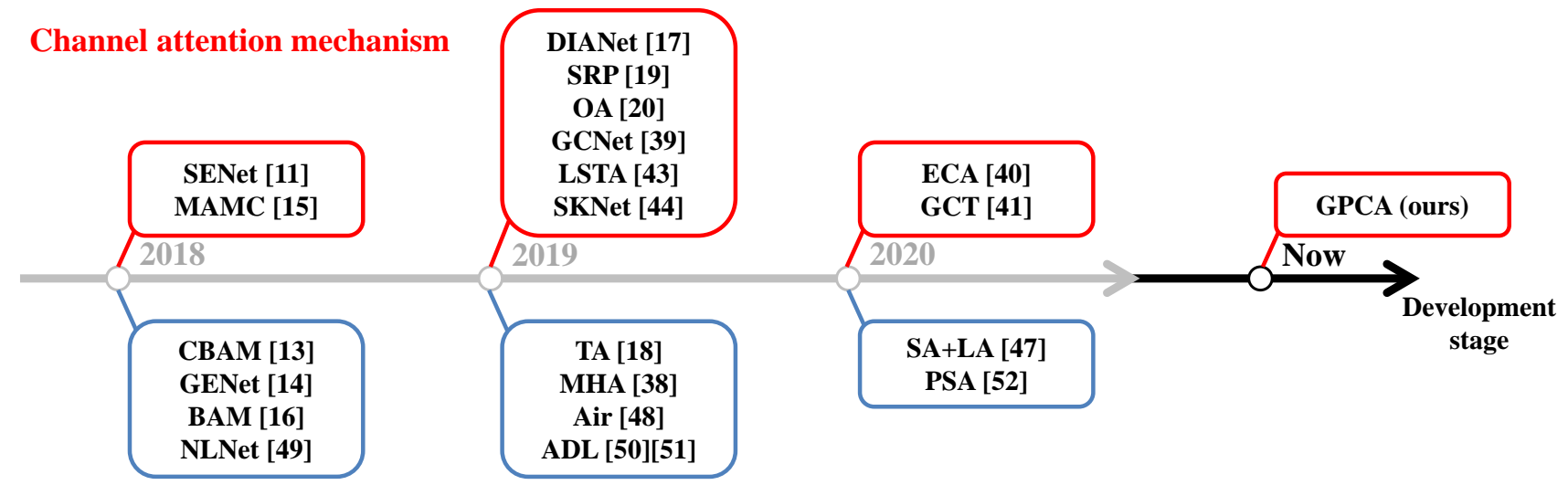

Spatial attention mechanism

Fig. 2: Development stages of attention mechanisms for visual tasks in recent years. The traditional attention mechanisms are commonly based on convolutional operations and pooling, while the GPCA module is derived within the probabilistic framework.

[20] have proposed several attention modules in different implementation ways. However, the reasons why the conventional attention modules can learn the importance weights of the channels and/or regions are difficult to be explained, although the authors of those papers described their own understandings of their proposed modules, respectively. One way for promoting the explainability of attention mechanisms is to design an attention mechanism under the probabilistic framework, since it is well-known in its mechanism's intuitiveness and reasonableness [21].

In this paper, we propose a novel attention mechanism, namely Gaussian process embedded channel attention (GPCA), to clarify the channel attention mechanism intuitively and reasonably in a probabilistic way. The graphical model of the GPCA is shown in Figure 1. Generally speaking, the channel attention masks are bounded in the interval $[0,1]$, which is a common boundary for traditional attentions. Hence, it is intuitive that we assume these attention mask values are generated from beta distributions. However, the beta distribution cannot be directly integrated into the endto-end training of the CNNs with an analytically tractable (closed-form) solution. The reason is that we need to find a differentiable function in terms of the distribution parameters for backpropagation. When we apply the beta variable in the CNN training, such differentiable function is infeasible due to the gamma function (defined by integration of the variable) in beta probability density function (PDF). To tackle the above difficulty, we utilize an appropriate approximation of the beta distribution to solve this problem. We name this approximation as "Sigmoid-Gaussian approximation", in which the Gaussian distributed variables can be transferred and bounded into the interval $[0,1]$ by a Sigmoid function. Given that a channel attention module learns the weights of channels by computing their correlations precisely within end-to-end training, Gaussian process (GP) [21], [22], which is a popular Bayesian learning framework, is integrated into the GPCA module as the prior of the Gaussian distributed variables. This aims to learn the correlations among different channels in an explicit way. In this case, the informative channels can be emphasized by the large values from the attention masks, extracted by the GPCA module.

Experimental results show that the proposed GPCA module can achieve the state-of-the-art performance on five datasets for the task of image classification. Meanwhile, we further evaluate the GPCA module in weakly supervised object localization (WSOL), object detection, and semantic segmentation tasks and demonstrate its reliable state-of-theart performance.

\section{Related Work}

Attention mechanisms are well-known and play important roles in various visual tasks including image retrieval [23], [24], object detection [25], [26], [27], person re-identification [28], [29], [30], visual tracking [31], image super-resolution [32], [33], [34], semantic segmentation [35], [36], [37], and especially in image classification task [11], [13], [14], [15], [16], |17], [18], [19], [20], |38]. Most of the aforementioned works discuss what is the most effective way to introduce the attention mechanisms. These researches mainly concentrate on forcing CNNs to focus on the informative channels and/or the regions with convolutional feature maps. In general, the aforementioned work can be divided into three categories, i.e., channel attention mechanisms, spatial attention mechanisms, and channelspatial attention mechanisms. Usually, the spatial attention is combined with the channel attention in the $\mathrm{CNN}$ frameworks to further regularize the feature maps. Hence, we discuss the spatial and the channel-spatial attention mechanisms together in Section 2.2. We show the development stages of the attention mechanisms in Figure 2

\subsection{Channel Attention Mechanism}

Squeeze-and-excitation network (SENet) [11], which has been widely used in various network architectures, was proposed to investigate the channel relationship by a 2-layer fully-connected (FC) network, yielding significant improvements in the image classification task. By proposing the squeeze-and-excitation (SE) mechanism, the SENet squeezes the feature maps across their spatial dimensions to introduce a channel descriptor and excites the embedded channel descriptor to per-channel modulation weights by a selfgating mechanism. The work in [15] was applied in CNNs, consisting of one-squeeze multi-excitation (OSME) modules and a multi-attention multi-class constraint (MAMC) for fine-grained image classification. The OSME modules 
are the extensions of the architecture of the SENet. They perform as channel attention modules for multiple discriminative regions discovered in the input images and improve the overall recognition performance. In addition, stochastic region pooling (SRP) [19], a channel attention module, randomly selected the square regions from the feature maps to extract the channel descriptors and forced them more representative and diverse. Then, it excited the channel descriptors in a similar way as the SENet to produce channel attention masks. Cao et al. [39] replaced the global average pooling (GAP) of the SENet by a context modeling to describe the global context and named it as the global context network (GCNet). Wang et al. [40] proposed a local cross-channel interaction strategy for channel attention mechanism to introduce a lightweight SENet and named it as efficient channel attention (ECA) module. Gated channel transformation (GCT) [41] is another variant of the SENet, which combines a global context embedding, a channel normalization, and a gating adaptation for efficient and accurate contextual information modeling.

There are still other attention-related works which import the channel attention mechanisms with special motivations. Dense-and-implicit attention network (DIANet) [17] introduced a modified long short term memory (LSTM) [42] model as the channel attention module for communication of the information among different convolutional layers and enhanced the generalization capacity of CNNs by repeatedly fixing the information. Sudhakaran et al. [43] directly integrated the attention mechanism into the LSTM for egocentric action recognition and named it as the long shortterm attention (LSTA). The work in |20| captured the channel attentions after each convolutional block for rectifying the output likelihood distributions of the whole $\mathrm{CNN}$, rather than the corresponding feature maps in the blocks. This can be considered as an output attention (OA) mechanism. In addition, the channel attention mechanism has also been utilized for convolutional kernel selection. Selective kernel network (SKNet) [44] was proposed to dynamically adjust the receptive field sizes of convolutional layers in CNNs by weighting the parallel convolutional kernels with different kernel sizes in a convolutional layer. Chen at al. [45] proposed a dynamic convolution with the SENet module generating the weights of the convolution kernels.

\subsection{Spatial Attention Mechanism}

Bottleneck attention module (BAM) |16| created two separate and parallel pathways, i.e., the channel-wise and the spatial-wise ones, to construct the channel and spatial attentions. It combined them together as an element-wise attention. Meanwhile, different from the BAM, the convolutional block attention module (CBAM) [13] inferred the elementwise attention maps by cascade-connected channels and spatial attentions for the adaptive refinement of the intermediate feature maps. Gather-excite network (GENet) [14] introduced a parametric gather-excite operator pair into the residual blocks in ResNet [2], which is considered as a generalization of the aforementioned SENet. It yielded further performance gains. The gathering operation aggregates the feature responses across the spatial neighbourhoods in a feature map, which is a two-dimensional pooling. The exciting operation redistributes the pooled information to local

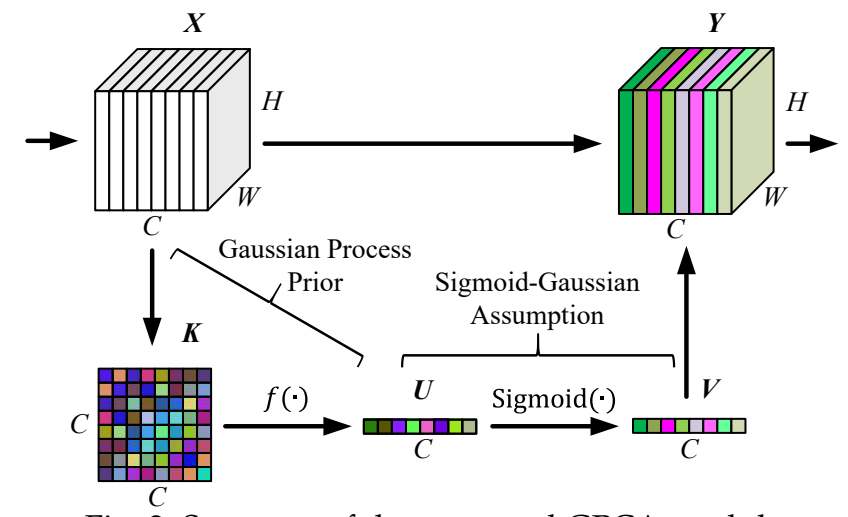

Fig. 3: Structure of the proposed GPCA module.

features via nearest neighbour interpolation. In addition, two-level attention (TA) module [18] implemented the channel and spatial attentions which characterized the objectlevel and the pixel-level attention, respectively. It combined these two attentions through a second-order response transform called bilinear pooling [46] for fine-grained visual recognition. Zheng et al. [47] proposed a global and local knowledge-aware attention network, combining a statisticbased attention (SA) and a learning-based attention (LA) together, for action recognition. Yang et al. [48] introduced an attention inspiring receptive-fields (Air) module in a encoder-decoder architecture and implemented it by convolutional layers and a bilinear pooling.

Self-attention [10] is a special (channel-)spatial attention mechanism, which yields the weighted averaged values computed from the feature maps themselves. Non-local neural network (NLNet) [49] combined a trilinear pooling with $1 \times 1$-convolutional layers and softmax functions to capture the long-range dependencies with deep neural networks in videos. Attention-based dropout layer (ADL) [50], [51] applied the self-attention mechanism by a channel-wise pooling and generated the spatial attention maps for weakly supervised object localization. Bello et al. [38] developed a two-dimensional relative self-attention mechanism which introduces multihead-attention (MHA) as a key component. Zhao et al. [52] explored variations of self-attention for image recognition and proposed a pairwise self-attention (PSA) network to improve the robustness and the generalization ability of the attention mechanism.

\section{Gaussian Process Embedded Channel At- TENTION (GPCA)}

In this section, we introduce the Gaussian process embedded channel attention (GPCA) module, for the purpose of modeling the channel attention mechanism in a probabilistic way and interpreting it intuitively and reasonably.

A GPCA module can learn the correlations between channels in CNNs via a Gaussian process [21] and calibrate the input feature maps by multiple channel-wise masks. Formally, we define the input feature maps by $\boldsymbol{X} \in R^{C \times W \times H}$ as shown in Figure 3. where $C, W$, and $H$ are the number of channels, the width, and the height of the feature maps, respectively. Meanwhile, the final output $\boldsymbol{Y} \in R^{C \times W \times H}$ of the GPCA module is obtained by scaling $\boldsymbol{X}$ with the attention mask vector $\boldsymbol{V}=\left[v_{1}, \cdots, v_{C}\right]^{\mathrm{T}}$ as

$$
\boldsymbol{y}_{c}=v_{c} \cdot \boldsymbol{x}_{c}, c=1, \cdots, C,
$$

where $\boldsymbol{x}_{c}$ and $\boldsymbol{y}_{c}$ are the channels of $\boldsymbol{X}$ and $\boldsymbol{Y}$, respectively. 

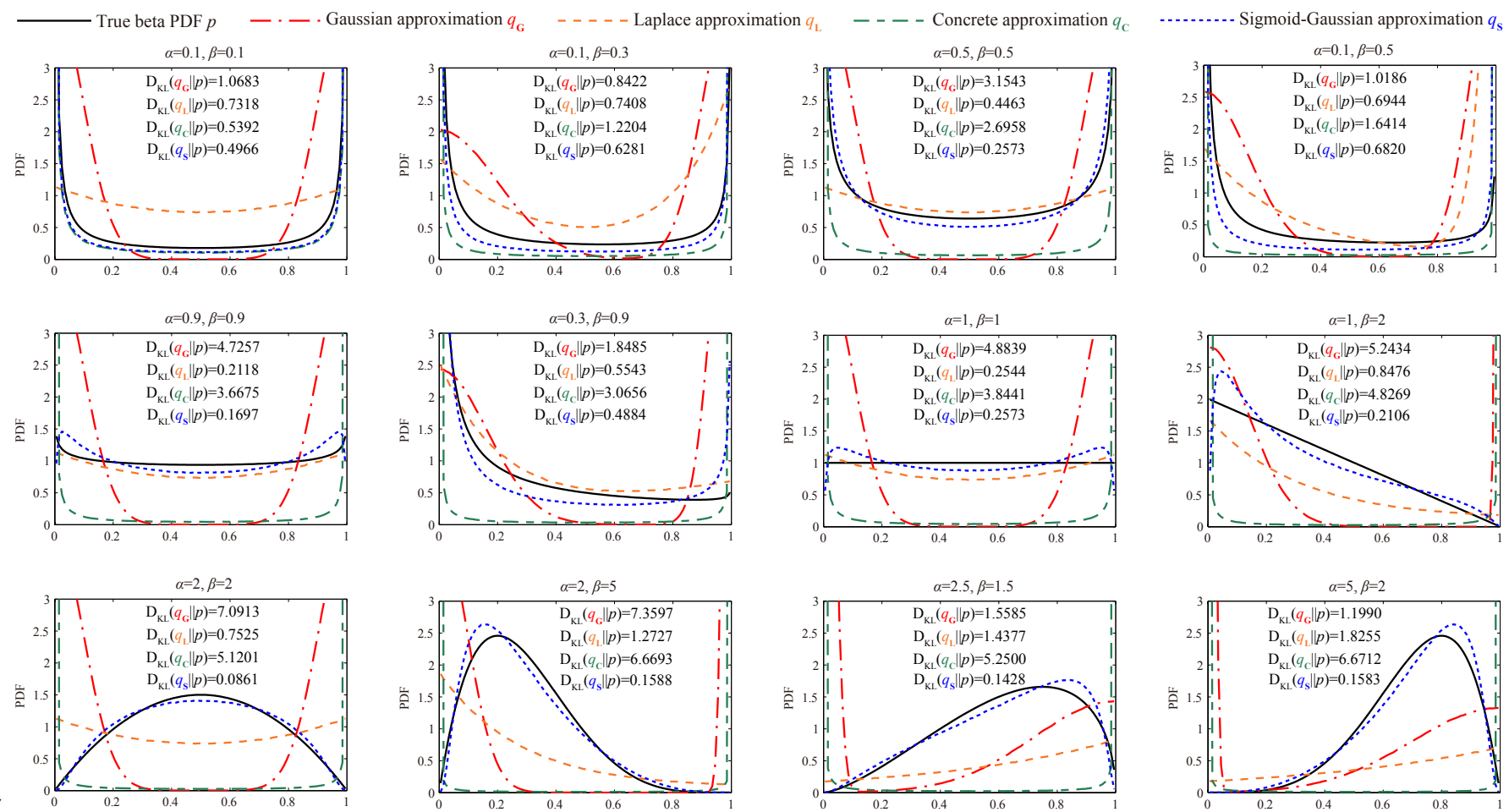

Fig. 4: Probability density function (PDF) comparisons of the proposed Sigmoid-Gaussian approximation $\left(q_{S}\right)$ with the beta distribution $(p)$, the Gaussian approximation $\left(q_{G}\right)$ and the Laplace approximation $\left(q_{L}\right)$ used in [53], and concrete approximation $\left(q_{C}\right)$ proposed in [54]. The Kullback-Leibler $(\mathrm{KL})$ divergences are calculate from all the approximations to their corresponding true beta PDFs with different $\alpha$ and $\beta$.

\subsection{Attention Modeling via Sigmoid-Gaussian Approxi- mation}

The element $v_{c}$ of the attention mask vector $\boldsymbol{V}$ is commonly set as a [0,1]-bounded variable [11], [13], [14], |16| to scale the input feature maps with the normalized importance weights. Thus, we assume that the elements in $\boldsymbol{V}$ follow beta distributions to represent the importance of the channels. The probability density function (PDF) of the beta distribution is defined as

$$
\operatorname{Beta}(x ; \alpha, \beta)=\frac{x^{\alpha-1}(1-x)^{\beta-1}}{\mathrm{~B}(\alpha, \beta)},
$$

where $\mathrm{B}(\alpha, \beta)=\frac{\Gamma(\alpha) \Gamma(\beta)}{\Gamma(\alpha+\beta)}$ and $\Gamma(\cdot)$ is the Gamma function. The beta distribution, as a member of the exponential family, is defined in a bounded interval, i.e., $[0,1]$, which is consistent with the definition of the channel attention. Meanwhile, the beta distribution is used for describing the statistical behavior of the percentages or the proportions in Bayesian learning such as certainty or importance. This further satisfies the principles of the attention modeling. In addition, it has flexible shapes (i.e., bell, " $\mathrm{U}$ ", or uniform shapes) depending on different values of the parameters $\alpha$ and $\beta$, which makes it flexible for the channel attention learning in general.

However, the beta distribution cannot be directly applied in the CNN training [53|. This is because when we apply the beta variable in the CNN training (normally using gradient descent-based algorithms), it is required to find a differentiable function $g(\cdot)$ for the backpropagation. With the beta PDF in (2), we have to compute the derivatives of the logarithm of the Gamma functions during the backpropagation. As the Gamma function $\Gamma(\cdot)$ is defined by

$$
\Gamma(x)=\int_{0}^{\infty} z^{x-1} e^{-z} d z,
$$

where the derivative of the logarithm of the Gamma function (i.e., the digamma function) is again defined by an integration form according to the Leibniz integral rule. Since $g(\cdot)$ contains the derivative of the logarithm of the Gamma function, it is not feasible to find a closed-form function to calculate $g(\cdot)$ explicitly. Although we can apply some numerical sampling methods, e.g., Markov chain Monte Carlo (MCMC), to numerically simulate $g(\cdot)$, the computational cost will be high, especially when dealing with high-dimensional data. In other words, we cannot directly employ the beta distribution in the CNN training, as the closed-form solution for $g(\cdot)$ is unavailable. Alternatively, we introduce an approximation $q(\alpha, \beta)$, named as SigmoidGaussian approximation, for the beta distribution to solve the unfeasible issue in backpropagation. A random variable $v \sim q(\alpha, \beta)$ following the distribution assumption can be generated by transferring a Gaussian distributed variable $u \sim \mathcal{N}\left(\mu, \sigma^{2}\right)$ as

$$
v=\operatorname{Sigmoid}(u)=\frac{1}{1+e^{-u}},
$$

and $u=\ln v-\ln (1-v)$. In this case, parameters $\alpha$ and $\beta$ can be obtained by matching the first- and second-order moments between $u$ and $v$ as

$$
\left\{\begin{array}{l}
\mu=\phi(\alpha)-\phi(\beta) \\
\sigma^{2}=\phi_{1}(\alpha)+\phi_{1}(\beta)
\end{array}\right.
$$

where $\phi(\cdot)$ and $\phi_{1}(\cdot)$ are the digamma and trigamma functions, respectively $[1]$ For better understanding, we derive the

1. Please refer to https://en.wikipedia.org/wiki/Beta_distribution 
equations in (5) by

$$
\begin{aligned}
\mu & =\mathrm{E}[u]=\mathrm{E}[\ln v-\ln (1-v)]=\mathrm{E}[\ln v]-\mathrm{E}[\ln (1-v)] \\
& =(\phi(\alpha)-\phi(\alpha+\beta))-(\phi(\beta)-\phi(\alpha+\beta))=\phi(\alpha)-\phi(\beta), \\
\sigma^{2} & =\operatorname{Var}[u]=\operatorname{Var}[\ln v-\ln (1-v)]=\operatorname{Var}\left[\ln \left(\frac{v}{1-v}\right)\right] \\
& =-\operatorname{Cov}\left[\ln \left(\frac{v}{1-v}\right), \ln \left(\frac{1-v}{v}\right)\right]=\phi_{1}(\alpha)+\phi_{1}(\beta),
\end{aligned}
$$

with the properties of the beta distribution. Since (6) and (7) illustrate a one-to-one mapping between $(\alpha, \beta)$ and $(\mu$, $\sigma)$, we can optimise $(\mu, \sigma)$ instead of $(\alpha, \beta)$ to facilitate backpropagation training.

For $v$ in (4), with the principle of integration by substitution, we have

$$
\begin{aligned}
\int p(u) d u & =\int \frac{1}{\sqrt{2 \pi} \sigma} e^{-\frac{(\ln v-\ln (1-v)-\mu)^{2}}{2 \sigma^{2}}} \cdot\left(\frac{1}{v(1-v)}\right) d v \\
& =\int p(v) d v \\
& =1 .
\end{aligned}
$$

To demonstrate the effectiveness of the SigmoidGaussian approximation, we conducted several groups of simulations with different parameters, as shown in Figure 4 We compare the PDF $q_{S}$ of the Sigmoid-Gaussian approximation with three different approximation methods (including Gaussian approximation $q_{G}$ and Laplace approximation $q_{L}$ used in [53], and concrete approximation $q_{C}[54]$ ) and the true beta PDFs $p$ with parameters $\alpha$ and $\beta$. The beta approximation methods can be divided into two groups, i.e., single distributions (including Gaussian approximation and Laplace approximation) and distributions via variable transfer (including concrete approximation and the proposed Sigmoid-Gaussian approximation). The proposed SigmoidGaussian approximation can approach the shapes (e.g., bell, " $U$ ", skewed bell shapes) of the true beta distribution with different parameters and has the same interval (i.e., $v \in[0,1])$ with a beta distributed variable. This demonstrates the proposed Sigmoid-Gaussian approximation can be a beta approximation solution. In addition, the KullbackLeibler (KL) divergences from the approximated PDFs $q_{S}$, $q_{G}, q_{L}$, or $q_{C}$ to the true beta PDFs $p$ are calculated with parameters $\alpha$ and $\beta$ in each group. It can be observed that $q_{S}$ yields more accurate approximation to the corresponding $p$ with smaller KL divergences. This clearly demonstrates the effectiveness of the Sigmoid-Gaussian approximation.

\subsection{Channel Correlation Modeling via GP Prior}

In order to adaptively model the correlations between channels, we introduce a Gaussian process $(\mathrm{GP})$ prior $\mathcal{G P}(\cdot)$ with the input feature map $\boldsymbol{X}$ for $\boldsymbol{U}$ as $\boldsymbol{U} \sim \mathcal{G P}(\boldsymbol{X})$. Here we treat the channels in $\boldsymbol{X}$ as the samples in $\mathcal{G} \mathcal{P}(\cdot)$. According to the definition of the Sigmoid-Gaussian assumption for beta approximation in Section 3.1. for the $c^{t h}$ channel, the parameters $\alpha_{c}$ and $\beta_{c}$ of the beta distribution can be matched by $\mu_{c}$ and $\sigma_{c}^{2}$ in (5) and we can treat the Gaussian processing as the prior of the parameters in the beta distribution.

Although we cannot obtain any exact targets of attention masks in CNNs, we are able to learn the channel correlations in $\boldsymbol{X}$ by $\boldsymbol{a}_{c} \in R^{1 \times(C-1)}$ for the $c^{t h}$ channel to others, which is defined as

$$
\boldsymbol{a}_{c}=\boldsymbol{K}_{c, i \neq c}\left(\boldsymbol{K}_{i \neq c, i \neq c}+\delta^{-1} \boldsymbol{I}_{C-1}\right)^{-1},
$$

where $\delta$ represents the precision of the Gaussian noise in the Gaussian process. For the purpose of adapting noninformative prior, we set it as a large number $\left(e . g ., 1 \times 10^{6}\right)$ in practice. $\boldsymbol{I}_{C-1}$ is an $(C-1) \times(C-1)$ identity matrix. $\boldsymbol{K}_{c, i \neq c} \in R^{1 \times(C-1)}$ and $\boldsymbol{K}_{i \neq c, i \neq c} \in R^{(C-1) \times(C-1)}$ are parts of the Gram matrix $\boldsymbol{K} \in R^{C \times C}$ in the Gaussian process [21], [22] with elements $K_{c, c^{\prime}}, c, c^{\prime}=1, \cdots, C$, defined as

$$
\begin{aligned}
K_{c, c^{\prime}} & =k\left(\boldsymbol{x}_{c}, \boldsymbol{x}_{c^{\prime}}\right) \\
& =\underbrace{\theta_{0} e^{-\theta_{1}\left\|\boldsymbol{x}_{c}-\boldsymbol{x}_{c^{\prime}}\right\|^{2}}}_{\text {Gaussian kernel }}+\underbrace{\theta_{2}}_{\text {Bias }}+\underbrace{\theta_{3} \boldsymbol{x}_{c} \boldsymbol{x}_{c^{\prime}}^{\mathrm{T}}}_{\text {Linear kernel }},
\end{aligned}
$$

where $k\left(\boldsymbol{x}_{c}, \boldsymbol{x}_{c^{\prime}}\right)$ is a kernel function with nonnegative parameters $\boldsymbol{\Theta}=\left\{\theta_{0}, \theta_{1}, \theta_{2}, \theta_{3}\right\}$ and consists of a Gaussian kernel, a linear kernel, and a bias. In practice, we can either have $\Theta$ fixed or set $\theta_{i}=e^{\tilde{\theta}_{i}}$ and optimize $\tilde{\theta}_{i}$ to satisfy the nonnegative constraints. In this paper, $\theta_{i}$ is implicitly optimized by $\tilde{\theta}_{i}$ in model training.

Then, we assume that the importance weight vector $\boldsymbol{U}=$ $\left[u_{1}, \cdots, u_{C}\right]^{\mathrm{T}}$ in Figure 3 that the $c^{t h}$ channel $u_{c}, c=$ $1, \cdots, C$, follows Gaussian distribution as

$$
u_{c} \sim \mathcal{N}\left(A_{c}, B_{c}\right),
$$

where $A_{c}, B_{c}$ are the mean and the variance of $u_{c}$, respectively. According to [21], they can be calculated as

$$
\begin{aligned}
A_{c} & =\frac{1}{C-1} \sum_{c^{\prime}=1, c^{\prime} \neq c}^{C} \tilde{a}_{c^{\prime}, c}, \\
B_{c} & =K_{c, c}-\boldsymbol{a}_{c} \boldsymbol{K}_{c, i \neq c}^{\mathrm{T}},
\end{aligned}
$$

where the row vector $\tilde{a}_{c} \in R^{1 \times C}$ is obtained by inserting a constant before index $c$ of the row $a_{c}$ as

$$
\tilde{a}_{c, i}=\left\{\begin{array}{ll}
a_{c, i} & 1 \leq i<c \\
\epsilon & i=c \\
a_{c, i-1} & c<i \leq C
\end{array},\right.
$$

where $\epsilon$ is a constant. Note that the padding constant $\epsilon$ in (14) is for clearly clarifying the correspondence of the columns in each row, as $a_{c, i}$ corresponds to channel $i$ when $1 \leq i<c, a_{c, i-1}$ corresponds to channel $i$ when $c<i \leq C$, and no elements in $a_{c}$ correspond to channel $c$. In (12), $\tilde{a}_{c, c}$ is not involved in the calculation of $A_{c}$, which means that the paddings can use any value and we opt to use zeros here for the purpose of simplification in calculation.

As $\boldsymbol{a}_{c}$ for each channel is calculated one-by-one in practice, we can obtain the correlations between any channel and the rest ones. Then, these correlations are averaged as the expected values of the channel attention weights, which is shown in (12). With the aforementioned one-by-one strategy, the prior of each channel is set as a one-dimension Gaussian distribution. To specify, we fix $\boldsymbol{K}$ (during each iteration $\boldsymbol{K}$ is not updated when calculating $\boldsymbol{a}_{c}$ with different $c$ ) and consider each channel as the theoretical "test input" of the Gaussian process. Meanwhile, the other channels are considered as the "training inputs" of the Gaussian process.

In practice, the expectation of the random variable $v_{c}$ is considered as the final GPCA mask of the $c^{t h}$ channel, which is calculated by [21]

$$
\mathrm{E}\left[v_{c}\right] \approx \operatorname{Sigmoid}\left(\frac{A_{c}}{\sqrt{1+\frac{\pi}{8} B_{c}}}\right) .
$$

The algorithm of the GPCA is summarized in Algorithm 1 


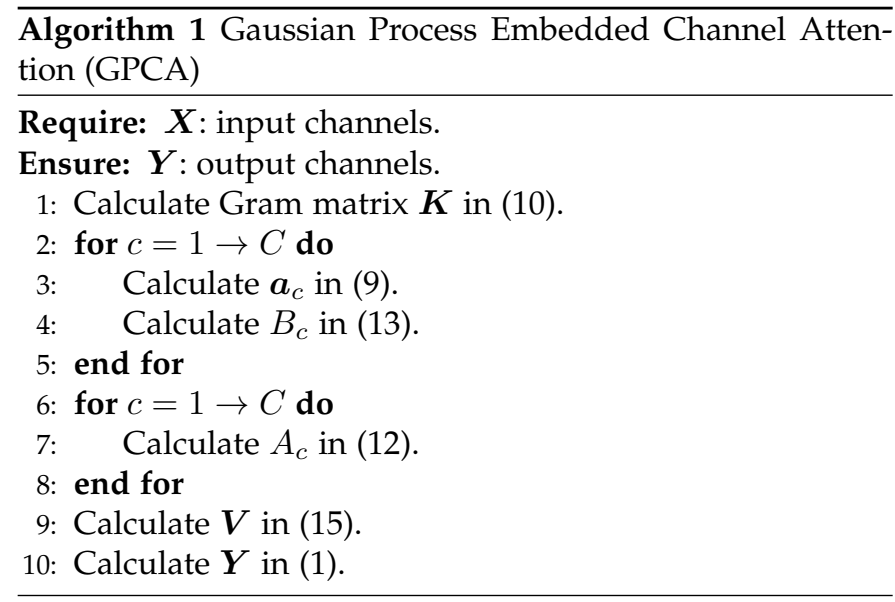

\subsubsection{Derivation of GP for Channel Correlation Modeling}

We are motivated by the assumption that modeling the channel correlation and then calculating the channel attention masks is equivalent to a GP for regression with pseudo targets. Here, the pseudo targets are based on the aspiration that the channel attention mechanism can reflect the actual correlation of each channel and reconstruct the pseudo target of one channel according to those of the other channels and the corresponding correlations.

Specifically, taking the $c^{\text {th }}$ channel $\boldsymbol{x}_{c}$ as a test sample with its pseudo target $z_{c}$, we define the pseudo targets of the other $(C-1)$ channels as $\boldsymbol{z}_{i \neq c}$. Here, we can transform $z_{c}$ to a vector $\boldsymbol{z}_{c}$ and set $\boldsymbol{z}_{c}=\boldsymbol{x}_{c}$, for the purpose of transforming the general regression task we defined into an autoregression one. In this case, reconstructing one channel with all the others can be considered as modeling the channel correlation and then calculating the channel attention masks. Since we can independently analyze each dimension of the vector $\boldsymbol{z}_{c}$, we show the derivation of the GP in terms of scalar $z_{c}$ in the following paragraphs for better demonstration. We further design an aggregation function $\Phi\left(\boldsymbol{X}_{i \neq c}\right) \in R^{M \times(C-1)}$ to map $\boldsymbol{X}_{i \neq c}$ into an $M$-dimensional feature space. Here, the relationship between the kernel function $K$. . in $(10)$ and the aggregation function $\Phi(\cdot)$ is as $K_{c, c^{\prime}}=k\left(\boldsymbol{x}_{c}, \boldsymbol{x}_{c^{\prime}}\right)=\boldsymbol{\Phi}\left(\boldsymbol{x}_{c}\right)^{\mathrm{T}} \boldsymbol{\Phi}\left(\boldsymbol{x}_{c^{\prime}}\right)=\boldsymbol{\Phi}_{c}^{\mathrm{T}} \boldsymbol{\Phi}_{c^{\prime}}$. Then, the relation between $\boldsymbol{z}_{i \neq c} \in R^{(C-1) \times 1}$ and $\Phi\left(\boldsymbol{X}_{i \neq c}\right)$ with parameters $\boldsymbol{w}_{c} \in R^{M \times 1}$ is

$$
\boldsymbol{z}_{i \neq c}=\underbrace{\Phi\left(\boldsymbol{X}_{i \neq c}\right)^{\mathrm{T}} \boldsymbol{w}_{c}}_{f\left(\boldsymbol{X}_{i \neq c}\right)}+\zeta \mathbf{1}_{C-1},
$$

where $f(\cdot)$ is the function for regression, the elements in the parameter vector $\boldsymbol{w}_{c}$ follow independent standard normal distributions, the noise term $\zeta$ follows a Gaussian distribution with zero mean and $\delta$ precision, and $\mathbf{1}_{C-1}$ is a $(C-1) \times 1$ vector that all the elements equal one. Then, $z_{c}$ is predicted by $f\left(\boldsymbol{x}_{c}\right)$ in the test phase as

$$
z_{c}=\underbrace{\Phi\left(\boldsymbol{x}_{c}\right)^{\mathrm{T}} \boldsymbol{w}_{c}}_{f\left(\boldsymbol{x}_{c}\right)}+\zeta .
$$

To estimate $\boldsymbol{w}_{c}$, the likelihood is calculated as

$$
p\left(\boldsymbol{z}_{i \neq c} \mid \boldsymbol{X}_{i \neq c}, \boldsymbol{w}_{c}\right)=\mathcal{N}\left(\boldsymbol{\Phi}^{\mathrm{T}} \boldsymbol{w}_{c}, \delta^{-1} \boldsymbol{I}_{\boldsymbol{C}-\mathbf{1}}\right),
$$

where $\boldsymbol{\Phi}$ is the shorthand of $\Phi\left(\boldsymbol{X}_{i \neq c}\right)$. According to the Bayes' rule, the posterior distribution $p\left(\boldsymbol{w}_{c} \mid \boldsymbol{X}_{i \neq c}, \boldsymbol{z}_{i \neq c}\right)$ is proportional to the product of the likelihood and the prior distribution of $\boldsymbol{w}_{c}$, which can be denoted as

$$
\begin{aligned}
& p\left(\boldsymbol{w}_{c} \mid \boldsymbol{X}_{i \neq c}, \boldsymbol{z}_{i \neq c}\right) \\
& \propto \exp \left(-\frac{\delta}{2}\left(\boldsymbol{z}_{i \neq c}-\boldsymbol{\Phi}^{\mathrm{T}} \boldsymbol{w}_{c}\right)^{\mathrm{T}}\left(\boldsymbol{z}_{i \neq c}-\boldsymbol{\Phi}^{\mathrm{T}} \boldsymbol{w}_{c}\right)\right) \cdot \exp \left(-\frac{1}{2} \boldsymbol{w}_{c}^{\mathrm{T}} \boldsymbol{w}_{c}\right) \\
& \propto \exp \left(-\frac{1}{2}\left(\boldsymbol{w}_{c}-\overline{\boldsymbol{w}}_{c}\right)^{\mathrm{T}}\left(\delta \boldsymbol{\Phi} \boldsymbol{\Phi}^{\mathrm{T}}+\boldsymbol{I}_{M}\right)\left(\boldsymbol{w}_{c}-\overline{\boldsymbol{w}}_{c}\right)\right),
\end{aligned}
$$

where $\overline{\boldsymbol{w}}_{c}=\delta\left(\delta \boldsymbol{\Phi} \boldsymbol{\Phi}^{\mathrm{T}}+\boldsymbol{I}_{M}\right)^{-1} \boldsymbol{\Phi} \cdot \boldsymbol{z}_{i \neq c}$. In this case, the posterior distribution $p\left(\boldsymbol{w}_{c} \mid \boldsymbol{X}_{i \neq c}, \boldsymbol{z}_{i \neq c}\right)$ is

$$
\begin{aligned}
& p\left(\boldsymbol{w}_{c} \mid \boldsymbol{X}_{i \neq c}, \boldsymbol{z}_{i \neq c}\right) \\
& =\mathcal{N}\left(\delta\left(\delta \boldsymbol{\Phi} \boldsymbol{\Phi}^{\mathrm{T}}+\boldsymbol{I}_{M}\right)^{-1} \boldsymbol{\Phi} \cdot \boldsymbol{z}_{i \neq c},\left(\delta \boldsymbol{\Phi} \boldsymbol{\Phi}^{\mathrm{T}}+\boldsymbol{I}_{M}\right)^{-1}\right) .
\end{aligned}
$$

Then, we can predict the pseudo target $\boldsymbol{z}_{c}$ of the $c^{t h}$ channel by $\boldsymbol{x}_{c}$ according to $\left\{\boldsymbol{x}_{i}, z_{i}\right\}_{i \neq c}$ as

$$
\begin{aligned}
f\left(\boldsymbol{x}_{c}\right) & =\boldsymbol{\Phi}_{c}^{\mathrm{T}} \boldsymbol{w}_{c} \\
& \sim \mathcal{N}(\underbrace{\boldsymbol{\Phi}_{c}^{\mathrm{T}} \delta\left(\delta \boldsymbol{\Phi} \boldsymbol{\Phi}^{\mathrm{T}}+\boldsymbol{I}_{M}\right)^{-1} \boldsymbol{\Phi}}_{\boldsymbol{a}_{c}} \cdot \boldsymbol{z}_{i \neq c}, \underbrace{\boldsymbol{\Phi}_{c}^{\mathrm{T}}\left(\delta \boldsymbol{\Phi} \boldsymbol{\Phi}^{\mathrm{T}}+\boldsymbol{I}_{M}\right)^{-1} \boldsymbol{\Phi}_{c}}_{B_{c}}),
\end{aligned}
$$

where $\boldsymbol{\Phi}_{c}$ is the shorthand of $\Phi\left(\boldsymbol{x}_{c}\right) \in R^{M \times 1}$. Here, $\boldsymbol{a}_{c}$ can model the relation between channels, since we obtain the mean of the (pseudo) prediction of the $c^{t h}$ channel by weighted summation $\boldsymbol{z}_{i \neq c}$ by weights $\boldsymbol{a}_{c}$. Meanwhile, $B_{c}$ can be considered as the variance of the channel attentions, when transforming $z_{c}$ to a vector $\boldsymbol{z}_{c}$ and setting $\boldsymbol{z}_{c}=\boldsymbol{x}_{c}$. In other words, we explicitly model the estimator $\mathrm{E}\left[\boldsymbol{x}_{c} \mid \boldsymbol{X}_{i \neq c}\right]$ that reconstructures $\boldsymbol{x}_{c}$ by $\boldsymbol{X}_{i \neq c}$ to compute the optimal attention values (i.e., importance weights) by following the certain criterion of the GP.

Here, we will derive the expressions of $\boldsymbol{a}_{c}$ and $B_{c}$ in (21), respectively, as those given by (9) and (13). Denote $\kappa=$ $\delta \boldsymbol{\Phi} \boldsymbol{\Phi}^{\mathrm{T}}+\boldsymbol{I}_{M}$, we have

$$
\boldsymbol{\kappa} \boldsymbol{\Phi}=\left(\delta \boldsymbol{\Phi} \boldsymbol{\Phi}^{\mathrm{T}}+\boldsymbol{I}_{M}\right) \boldsymbol{\Phi}=\delta \boldsymbol{\Phi} \underbrace{\left(\boldsymbol{\Phi}^{\mathrm{T}} \boldsymbol{\Phi}+\delta^{-1} \boldsymbol{I}_{C-1}\right)}_{\boldsymbol{\tau}} .
$$

Following the principals of matrix algebra, it can be obtained that

$$
\boldsymbol{\kappa}^{-1} \boldsymbol{\kappa} \mathbf{\Phi} \boldsymbol{\tau}^{-1}=\boldsymbol{\kappa}^{-1} \delta \boldsymbol{\Phi} \boldsymbol{\tau} \boldsymbol{\tau}^{-1} \Longrightarrow \mathbf{\Phi} \boldsymbol{\tau}^{-1}=\boldsymbol{\kappa}^{-1} \delta \boldsymbol{\Phi} .
$$

With (23), $\boldsymbol{a}_{c}$ can be reformulated as

$$
\begin{aligned}
\boldsymbol{a}_{c} & =\boldsymbol{\Phi}_{c}^{\mathrm{T}}\left(\boldsymbol{\kappa}^{-1} \delta \boldsymbol{\Phi}\right)=\boldsymbol{\Phi}_{c}^{\mathrm{T}}\left(\boldsymbol{\Phi} \boldsymbol{\tau}^{-1}\right) \\
& =\underbrace{\boldsymbol{\Phi}_{c}^{\mathrm{T}} \boldsymbol{\Phi}}_{\boldsymbol{K}_{c, i \neq c}}(\underbrace{\boldsymbol{\Phi}^{\mathrm{T}} \boldsymbol{\Phi}}_{\boldsymbol{K}_{i \neq c, i \neq c}}+\delta^{-1} \boldsymbol{I}_{C-1})^{-1} .
\end{aligned}
$$

For the extension of $B_{c}$, we introduce the matrix inversion lemma (or called Woodbury, Sherman \& Morrison formula) as

$$
\begin{aligned}
& \left(\boldsymbol{Z}+\boldsymbol{U} \boldsymbol{W} \boldsymbol{V}^{\mathrm{T}}\right)^{-1} \\
& =\boldsymbol{Z}^{-1}-\boldsymbol{Z}^{-1} \boldsymbol{U}\left(\boldsymbol{W}^{-1}+\boldsymbol{V}^{\mathrm{T}} \boldsymbol{Z}^{-1} \boldsymbol{U}\right)^{-1} \boldsymbol{V}^{\mathrm{T}} \boldsymbol{Z}^{-1},
\end{aligned}
$$

where $\boldsymbol{Z}, \boldsymbol{U}, \boldsymbol{W}$, and $\boldsymbol{V}$ are matrices. Here, we set $\boldsymbol{Z}=\boldsymbol{I}_{M}$, $\boldsymbol{U}=\boldsymbol{V}=\boldsymbol{\Phi}$, and $\boldsymbol{W}=\delta \boldsymbol{I}_{C-1}$, respectively and obtain

$$
\begin{aligned}
B_{c} & =\boldsymbol{\Phi}_{c}^{\mathrm{T}}\left(\boldsymbol{\Phi}\left(\delta \boldsymbol{I}_{C-1}\right) \boldsymbol{\Phi}^{\mathrm{T}}+\boldsymbol{I}_{M}\right)^{-1} \boldsymbol{\Phi}_{c} \\
& =\boldsymbol{\Phi}_{c}^{\mathrm{T}}\left(\boldsymbol{I}_{M}-\boldsymbol{I}_{M} \boldsymbol{\Phi}\left(\delta^{-1} \boldsymbol{I}_{C-1}+\boldsymbol{\Phi}^{\mathrm{T}} \boldsymbol{I}_{M} \boldsymbol{\Phi}^{-1} \boldsymbol{\Phi}^{\mathrm{T}} \boldsymbol{I}_{M}\right) \boldsymbol{\Phi}_{c}\right. \\
& =\underbrace{\boldsymbol{\Phi}_{c}^{\mathrm{T}} \boldsymbol{\Phi}_{c}}_{K_{c, c}}-\underbrace{\boldsymbol{\Phi}_{c}^{\mathrm{T}} \boldsymbol{\Phi}\left(\boldsymbol{\Phi}^{\mathrm{T}} \boldsymbol{\Phi}+\delta^{-1} \boldsymbol{I}_{C-1}\right)^{-1}}_{\boldsymbol{a}_{c}} \underbrace{\boldsymbol{\Phi}^{\mathrm{T}} \boldsymbol{\Phi}_{c}}_{\boldsymbol{K}_{c, i \neq c}^{\mathrm{T}}} .
\end{aligned}
$$


TABLE 1: Statistics of image classification datasets including the class number, the training and test sample number, and the input image size.

\begin{tabular}{lcccc}
\hline Dataset & \#class & \#training & \#test & Image size \\
\hline Cifar-10 & 10 & 50,000 & 10,000 & $32 \times 32$ \\
Cifar-100 & 100 & 50,000 & 10,000 & $32 \times 32$ \\
miniImageNet & 100 & 50,000 & 10,000 & $224 \times 224$ \\
ImageNet-32 $\times 32$ & 1000 & $1,281,123$ & 50,000 & $32 \times 32$ \\
ImageNet & 1000 & $1,281,123$ & 50,000 & $224 \times 224$ \\
\hline
\end{tabular}

3.2.2 Derivation of Approximated Expectation of SigmoidGaussian Approximation

The expectation of the variable $v_{c}$ can be calculated by

$$
\begin{aligned}
\mathrm{E}\left[v_{c}\right] & =\int_{0}^{1} v_{c} q\left(v_{c}\right) d v_{c} \\
& =\int_{-\infty}^{+\infty} \operatorname{Sigmoid}\left(u_{c}\right) \mathcal{N}\left(u_{c} ; A_{c}, B_{c}\right) d u_{c} \\
& \approx \int_{-\infty}^{+\infty} \varphi\left(\sqrt{\frac{\pi}{8}} u_{c}\right) \mathcal{N}\left(u_{c} ; A_{c}, B_{c}\right) d u_{c} \\
& =\varphi\left(\frac{A_{c}}{\sqrt{\frac{8}{\pi}+B_{c}}}\right) \approx \operatorname{Sigmoid}\left(\frac{A_{c}}{\sqrt{1+\frac{\pi}{8} B_{c}}}\right),
\end{aligned}
$$

where

$$
\varphi(x)=\int_{-\infty}^{x} \mathcal{N}(t ; 0,1) d t \approx \operatorname{Sigmoid}(\lambda x)
$$

is the cumulative distribution function of a standard normal distribution (or called probit function) with equality condition as $x=0$ [55]. The weight $\lambda=\sqrt{\frac{8}{\pi}}$ can be obtained by matching the first-order derivatives between $\varphi(x)$ and Sigmoid $(\lambda x)$ at $x=0$. Then the approximated expectation in 28 is used for the final GPCA mask to the $c^{t h}$ channel.

\section{EXPERIMENTS ON IMAGE CLASSIFICATION}

In this section, we compare the proposed GPCA with eight recently proposed attention modules, i.e., SENet [11], NLNet [49], BAM [16], CBAM [13], SRP [19], OA [20], ECA [40], and GCT [41], on five datasets for image classification.

\subsection{Datasets}

The five image datasets includes Cifar-10/-100 [56], miniImageNet [57], ImageNet-32 × 32 [58], ImageNet [59] datasets. The summary of the datasets is listed in Table 1 In the miniImageNet dataset, 500 and 100 images of per class are respectively randomly selected from the full ImageNet dataset for training and test. The ImageNet- $32 \times 32$ dataset is more difficult than the ImageNet dataset and all the images are resized to $32 \times 32$ for training and test [58].

\subsection{Implementation Details}

For the Cifar-10 and the Cifar-100 datasets, VGG16 [1], ResNet50 [2], and DenseNet40 [3] models were used as the base models for the proposed GPCA and other referred modules. Adopting the stochastic gradient descent (SGD) optimizer, we trained each model 300 epochs with batch size of 256, and the initial learning rates were set as 0.1 and decayed by a factor of 10 at the $150^{t h}$ and the $225^{t h}$ epochs. The momentum and the weight decay values were kept as 0.9 and $5 \times 10^{-4}$, respectively. All the methods were conducted three times with random initialization and the means and the standard deviations of the classification accuracies are reported.
TABLE 2: The means and standard deviations of the accuracies $(\%)$ on the Cifar-10 and the Cifar-100 datasets, and the $p$-values $(p)$ of the Student's $t$-test between the

\begin{tabular}{|c|c|c|c|c|}
\hline Model & Cifar-10 & $p$-value & Cifar-100 & $p$-value \\
\hline VGG16 (baseline) & $93.90 \pm 0.10$ & * & $73.64 \pm 0.14$ & * \\
\hline VGG16+SENet (2018) & $94.04 \pm 0.09$ & * & $73.86 \pm 0.03$ & * \\
\hline VGG16+NLNet (2018) & $93.95 \pm 0.34$ & * & $73.86 \pm 0.07$ & * \\
\hline VGG16+CBAM-S (2018) & $\underline{94.12} \pm \underline{0.10}$ & * & $\underline{74.31} \pm \underline{0.06}$ & * \\
\hline VGG16+ECA (2020) & $\overline{94.11} \pm \overline{0.07}$ & * & $\overline{73.65} \pm \overline{0.18}$ & * \\
\hline VGG16+GCT (2020) & $94.12 \pm 0.12$ & * & $73.67 \pm 0.23$ & * \\
\hline VGG16+GPCA (ours) & $94.51 \pm 0.03$ & $\mathrm{~N} / \mathrm{A}$ & $74.65 \pm 0.01$ & N/A \\
\hline ResNet50 (baseline) & $93.52 \pm 0.05$ & * & $71.88 \pm 0.06$ & * \\
\hline ResNet50+SENet (2018) & $94.16 \pm 0.05$ & * & $72.64 \pm 0.05$ & * \\
\hline ResNet50+NLNet (2018) & $93.68 \pm 0.15$ & * & $71.31 \pm 1.65$ & * \\
\hline ResNet50+BAM (2018) & $94.14 \pm 0.04$ & * & $\underline{73.36} \pm \underline{0.09}$ & * \\
\hline ResNet50+CBAM (2018) & $94.06 \pm 0.03$ & * & $73.01 \pm 0.06$ & * \\
\hline ResNet50+CBAM-S (2018) & $94.00 \pm 0.05$ & * & $73.29 \pm 0.04$ & * \\
\hline ResNet50+SRP (2019) & $94.11 \pm 0.01$ & * & $72.25 \pm 0.14$ & * \\
\hline ResNet50+OA (2019) & $94.09 \pm 0.06$ & * & $72.73 \pm 0.10$ & * \\
\hline ResNet50+ECA (2020) & $93.92 \pm 0.21$ & * & $72.38 \pm 0.20$ & * \\
\hline ResNet50+GCT (2020) & $\underline{94.37} \pm \underline{0.25}$ & * & $72.91 \pm 0.11$ & * \\
\hline ResNet50+GPCA (ours) & $\overline{94.93} \pm \overline{0.01}$ & N/A & $73.93 \pm 0.02$ & N/A \\
\hline DenseNet40 (baseline) & $93.02 \pm 0.07$ & * & $71.47 \pm 0.07$ & * \\
\hline DenseNet40+SENet (2018) & $93.22 \pm 0.13$ & * & $72.52 \pm 0.05$ & * \\
\hline DenseNet40+NLNet (2018) & $93.47 \pm 0.22$ & * & $71.38 \pm 0.06$ & * \\
\hline DenseNet40+BAM (2018) & $\underline{93.63} \pm \underline{0.08}$ & * & $71.67 \pm 0.15$ & * \\
\hline DenseNet40+CBAM (2018) & $\overline{93.19} \pm \overline{0.14}$ & * & $71.93 \pm 0.07$ & * \\
\hline DenseNet40+CBAM-S (2018) & $93.43 \pm 0.18$ & * & $71.84 \pm 0.12$ & * \\
\hline DenseNet40+SRP (2019) & $93.20 \pm 0.06$ & * & $71.85 \pm 0.01$ & * \\
\hline DenseNet40+OA (2019) & $93.11 \pm 0.09$ & * & $71.61 \pm 0.09$ & * \\
\hline DenseNet40+ECA (2020) & $93.37 \pm 0.08$ & * & $72.13 \pm 0.06$ & * \\
\hline DenseNet40+GCT (2020) & $93.61 \pm 0.21$ & * & $72.41 \pm 0.22$ & * \\
\hline DenseNet40+GPCA (ours) & $94.16 \pm 0.03$ & N/A & $73.02 \pm 0.19$ & N/A \\
\hline DenseNet-BC100 (baseline) & $94.38 \pm 0.16$ & * & $76.99 \pm 0.25$ & * \\
\hline DenseNet-BC100+SENet (2018) & $93.80 \pm 0.07$ & * & $77.30 \pm 0.12$ & * \\
\hline DenseNet-BC100+NLNet (2018) & $94.61 \pm 0.24$ & * & $78.10 \pm 0.16$ & * \\
\hline DenseNet-BC100+BAM (2018) & $94.24 \pm 0.17$ & * & $78.15 \pm 0.31$ & * \\
\hline DenseNet-BC100+CBAM (2018) & $94.39 \pm 0.04$ & * & $77.54 \pm 0.34$ & * \\
\hline DenseNet40+CBAM-S (2018) & $94.49 \pm 0.10$ & * & $77.52 \pm 0.21$ & * \\
\hline DenseNet40+SRP (2019) & $94.53 \pm 0.08$ & * & $\underline{78.31} \pm \underline{0.21}$ & * \\
\hline DenseNet40+OA (2019) & $94.47 \pm 0.20$ & * & $77.05 \pm 0.05$ & * \\
\hline DenseNet-BC100+ECA (2020) & $\underline{94.66} \pm \underline{0.11}$ & * & $78.06 \pm 0.31$ & * \\
\hline DenseNet-BC100+GCT (2020) & $\overline{94.62} \pm \overline{0.01}$ & * & $78.27 \pm 0.21$ & * \\
\hline DenseNet-BC100+GPCA (ours) & $95.41 \pm 0.25$ & N/A & $78.81 \pm 0.18$ & N/A \\
\hline
\end{tabular}
accuracies of the proposed method and the other methods. Note that the best and the second best results of each base model are marked in bold and italic fonts, respectively. The significance level $\alpha$ is 0.05 . Notation: " "**: $p<0.05$.

For the miniImageNet and the ImageNet- $32 \times 32$ datasets, the VGG16, the ResNet18, and the ResNet34 models were used as the base models for all the methods. The settings of the optimizers and the initial learning rates were the same with the other two datasets. We trained each model 300 and 100 epochs with batch sizes of 128 and 256 on the two datasets, respectively. Meanwhile, the learning rate on the miniImageNet dataset was decayed by a factor of 10 at the $150^{t h}$ and the $225^{t h}$ epochs, which were the $50^{t h}$ and the $75^{\text {th }}$ epochs on the other dataset. All the methods were also conducted three times with random initialization and the means and the standard deviations of the classification accuracies are reported as well.

For the ImageNet dataset, the ResNet18 and the ResNet 50 models were used as the base models. The settings of the optimizers and the initial learning rates were the same with the other datasets. We trained for 100 epochs with batch sizes of 256. Meanwhile, the learning rate on the dataset was decayed by a factor of 10 at the $50^{t h}$ and the $75^{\text {th }}$ epochs. The proposed method was also conducted three times with random initialization and the means and the standard deviations of the classification accuracies are reported as well.

In addition, the two-sample Student's $t$-tests have been conducted between the accuracies of the proposed method and the other referred methods. We set the significance level $\alpha$ as 0.05 , i.e., statistically significant difference can be found 
TABLE 3: The means and standard deviations of test accuracies (acc., \%) on the miniImageNet dataset, and the $p$-values $(p)$ of the Student's $t$-test between the accuracies of the proposed method and the other methods. Note that the best and the second best results of each base model are marked in bold and italic fonts, respectively. The significance level $\alpha$ is 0.05 . Notation: "**": $p<0.05$.

\begin{tabular}{lcc}
\hline Model & Acc. & $p$-value \\
\hline VGG16 (baseline) & $81.36 \pm 0.08$ & $*$ \\
VGG16+SENet (2018) & $81.66 \pm 0.08$ & $*$ \\
VGG16+NLNet (2018) & $80.42 \pm 0.28$ & $*$ \\
VGG16+BAM (2018) & $81.38 \pm 0.04$ & $*$ \\
VGG16+CBAM (2018) & $81.42 \pm 0.13$ & $*$ \\
VGG16+CBAM-S (2018) & $81.62 \pm 0.04$ & $*$ \\
VGG16+SRP (2019) & $\underline{81.91} \pm \underline{0.13}$ & $*$ \\
VGG16+ECA (2020) & $81.74 \pm 0.15$ & $*$ \\
VGG16+GCT (2020) & $81.68 \pm 0.19$ & $*$ \\
VGG16+GPCA (ours) & $\mathbf{8 2 . 7 8} \pm \mathbf{0 . 0 1}$ & N/A \\
\hline ResNet18 (baseline) & $77.13 \pm 0.06$ & $*$ \\
ResNet18+SENet (2018) & $77.76 \pm 0.04$ & $*$ \\
ResNet18+NLNet (2018) & $77.64 \pm 0.19$ & $*$ \\
ResNet18+BAM (2018) & $77.87 \pm 0.03$ & $*$ \\
ResNet18+CBAM (2018) & $77.62 \pm 0.07$ & $*$ \\
ResNet18+CBAM-S (2018) & $\underline{78.02} \pm \underline{0.04}$ & $*$ \\
ResNet18+SRP (2019) & $77.93 \pm 0.06$ & $*$ \\
ResNet18+OA (2019) & $77.49 \pm 0.06$ & $*$ \\
ResNet18+ECA (2020) & $77.72 \pm 0.07$ & $*$ \\
ResNet18+GCT (2020) & $77.60 \pm 0.19$ & $*$ \\
ResNet18+GPCA (ours) & $\mathbf{7 8 . 8 7} \pm \mathbf{0 . 0 2}$ & N/A \\
\hline ResNet34 (baseline) & $78.08 \pm 0.12$ & $*$ \\
ResNet34+SENet (2018) & $78.44 \pm 0.02$ & $*$ \\
ResNet34+NLNet (2018) & $78.48 \pm 0.2$ & $*$ \\
ResNet34+BAM (2018) & $78.23 \pm 0.08$ & $*$ \\
ResNet34+CBAM (2018) & $78.16 \pm 0.10$ & $*$ \\
ResNet34+CBAM-S (2018) & $78.46 \pm 0.03$ & $*$ \\
ResNet34+SRP (2019) & $\underline{78.99} \pm \underline{0.03}$ & $*$ \\
ResNet34+OA (2019) & $78.14 \pm 0.04$ & $*$ \\
ResNet34+ECA (2020) & $78.68 \pm 0.14$ & $*$ \\
ResNet34+GCT (2020) & $78.17 \pm 0.12$ & $*$ \\
ResNet34+GPCA (ours) & $\mathbf{8 0 . 1 4} \pm \mathbf{0 . 0 2}$ & N/A \\
\hline
\end{tabular}

when $p$-value $(p)$ of the Student's $t$-test is smaller than 0.05 ; otherwise, there is no statistically significant difference.

All the attention modules were added in each residual block in the ResNets, but they were only applied after the last convolutional layer of the VGG16 model. Note that in a VGG16 model, the output size of feature maps of the last convolutional layer was $512 \times 1 \times 1$ while the size of input images was $3 \times 32 \times 32$ (i.e., the Cifar-10, the Cifar-100, and the ImageNet- $32 \times 32$ datasets), and the BAM, the CBAM, and the SRP cannot work in this case. However, the channel attention of the CBAM can be separately applied in a VGG16 model, named as CBAM-S. In addition, the OA needs to be used in multiple blocks and cannot be implemented in the VGG16 model on all the datasets either.

\subsection{Performance on Cifar-10/-100 Datasets}

From Table 2, the proposed GPCA module performs the best among all the methods with the VGG16, the ResNet50, and the DenseNet 40 models on both Cifar-10 and Cifar100 datasets, respectively. Firstly, on the Cifar-10 dataset, the proposed GPCA module achieves the classification accuracies at $94.51 \%, 94.93 \%$, and $94.16 \%$ based on the VGG16, the ResNet50, the DenseNet40 models, respectively. Meanwhile, the proposed GPCA module with different base models exceeds their baseline models, at about $0.6 \%, 1.4 \%$, $1.1 \%$ improvements, respectively. Moreover, the proposed
TABLE 4: The means and standard deviations of both top-1 and top- 5 test accuracies (acc., \%) on the ImageNet-32 $\times 32$ dataset, and the $p$-values $(p)$ of the Student's $t$-test between the accuracies of the proposed method and the other methods. Note that the best and the second best results of each base model are marked in bold and italic fonts, respectively. The significance level $\alpha$ is 0.05 . Notation: " ${ }^{* \prime \prime}: p<0.05$.

\begin{tabular}{lcc|cc}
\hline Model & Top-1 acc. & $p$-value & Top-5 acc. & $p$-value \\
\hline VGG16 (baseline) & $39.70 \pm 0.02$ & $*$ & $62.66 \pm 0.03$ & $*$ \\
VGG16+SENet (2018) & $39.82 \pm 0.11$ & $*$ & $62.99 \pm 0.10$ & $*$ \\
VGG16+NLNet (2018) & $41.39 \pm 0.22$ & $*$ & $64.11 \pm 0.18$ & $*$ \\
VGG16+CBAM-S (2018) & $39.56 \pm 0.08$ & $*$ & $62.84 \pm 0.06$ & $*$ \\
VGG16+ECA (2020) & $\underline{41.89} \pm \underline{0.06}$ & $*$ & $\underline{64.45} \pm \underline{0.18}$ & $*$ \\
VGG16+GCT (2020) & $41.79 \pm 0.03$ & $*$ & $\underline{64.45} \pm \underline{0.32}$ & $*$ \\
VGG16+GPCA (ours) & $\mathbf{4 2 . 5 8} \pm \mathbf{0 . 0 1}$ & N/A & $\mathbf{6 5 . 6 5} \pm \mathbf{0 . 0 7}$ & N/A \\
\hline ResNet18 (baseline) & $47.75 \pm 0.07$ & $*$ & $72.70 \pm 0.13$ & $*$ \\
ResNet18+SENet (2018) & $47.89 \pm 0.09$ & $*$ & $72.98 \pm 0.02$ & $*$ \\
ResNet18+NLNet (2018) & $47.6 \pm 0.43$ & $*$ & $72.53 \pm 0.27$ & $*$ \\
ResNet18+BAM (2018) & $47.90 \pm 0.07$ & $*$ & $73.08 \pm 0.03$ & $*$ \\
ResNet18+CBAM (2018) & $48.48 \pm 0.08$ & $*$ & $73.25 \pm 0.03$ & $*$ \\
ResNet18+CBAM-S (2018) & $48.31 \pm 0.13$ & $*$ & $73.37 \pm 0.09$ & $*$ \\
ResNet18+SRP (2019) & $\underline{48.84} \pm \underline{0.04}$ & $*$ & $\underline{73.74} \pm \underline{0.04}$ & $*$ \\
ResNet18+OA (2019) & $48.62 \pm 0.08$ & $*$ & $73.21 \pm 0.05$ & $*$ \\
ResNet18+ECA (2020) & $48.44 \pm 0.09$ & $*$ & $73.11 \pm 0.08$ & $*$ \\
ResNet18+GCT (2020) & $48.70 \pm 0.12$ & $*$ & $72.95 \pm 0.03$ & $*$ \\
ResNet18+GPCA (ours) & $\mathbf{4 9 . 5 8} \pm \mathbf{0 . 0 2}$ & N/A & $\mathbf{7 5 . 0 7} \pm \mathbf{0 . 0 6}$ & N/A \\
\hline ResNet34 (baseline) & $49.82 \pm 0.10$ & $*$ & $74.34 \pm 0.02$ & $*$ \\
ResNet34+SENet (2018) & $50.30 \pm 0.04$ & $*$ & $74.89 \pm 0.21$ & $*$ \\
ResNet18+NLNet (2018) & $49.46 \pm 0.13$ & $*$ & $74.65 \pm 0.32$ & $*$ \\
ResNet34+BAM (2018) & $\underline{50.70} \pm \underline{0.01}$ & $*$ & $74.79 \pm 0.03$ & $*$ \\
ResNet34+CBAM (2018) & $50.28 \pm 0.05$ & $*$ & $\underline{74.89} \pm \underline{0.03}$ & $*$ \\
ResNet34+CBAM-S (2018) & $50.65 \pm 0.09$ & $*$ & $75.07 \pm 0.08$ & $*$ \\
ResNet34+SRP (2019) & $50.24 \pm 0.10$ & $*$ & $74.73 \pm 0.07$ & $*$ \\
ResNet34+OA (2019) & $50.04 \pm 0.06$ & $*$ & $74.88 \pm 0.03$ & $*$ \\
ResNet34+ECA (2020) & $50.33 \pm 0.07$ & $*$ & $74.59 \pm 0.23$ & $*$ \\
ResNet34+GCT (2020) & $50.23 \pm 0.06$ & $*$ & $74.47 \pm 0.14$ & $*$ \\
ResNet34+GPCA (ours) & $\mathbf{5 1 . 2 4} \pm \mathbf{0 . 0 2}$ & N/A & $\mathbf{7 5 . 7 9} \pm \mathbf{0 . 0 4}$ & N/A \\
\hline & & & &
\end{tabular}

GPCA module with the VGG16 model outperforms the CBAM-S and the GCT module ( $94.12 \%$ of both), the two best referred methods, at about $0.4 \%$, while the proposed GPCA module with the ResNet50 model surpasses the GCT module with the ResNet50 model (94.37\%) more than $0.5 \%$. The proposed GPCA module with the DenseNet40 model also achieves more than $0.5 \%$ improvement than the BAM module with the DenseNet 40 model.

Similarly, on the Cifar-100 dataset, the proposed GPCA module achieves the classification accuracies at $74.65 \%$, $73.93 \%, 73.02 \%$ on the VGG16, the ResNet50, and the DenseNet 40 base models, respectively. It performs the best among all the methods as shown in Table 2 The GPCAbased VGG16, ResNet50, and DenseNet40 models have significant improvements compared with the corresponding base models at more than $1 \%, 2 \%$, and $1.5 \%$, respectively. In addition, they also gain about $0.3 \%, 0.6 \%$, and $0.5 \%$ improvements compared with the best referred models, i.e., the CBAM-S module with the VGG16 (74.31\%), the BAM module with the ResNet50 ( $73.36 \%)$, and the SENet module with the DenseNet40 (72.52\%) models, respectively.

According to the $p$-values of Student's $t$-test between the proposed method and the other methods in Table 2 , the results obtained by GPCA are all statistically significant.

\subsection{Performance on MinilmageNet Dataset}

In Table 3. the proposed GPCA module obtained the best results with VGG16, ResNet18 and ResNet34 models among the referred methods on the miniImageNet dataset, which achieves the classification accuracies at $82.78 \%, 78.87 \%$, and $80.14 \%$, respectively. Applying VGG16 as the base model, the classification accuracy of the GPCA with the VGG16 
TABLE 5: The means and standard deviations of both top-1 and top- 5 test accuracies (acc., \%) on the ImageNet dataset, and the $p$-values $(p)$ of the Student's $t$-test between the accuracies of the proposed method and the other methods. Note that the best and the second best results of each base model are marked in bold and italic fonts, respectively. The significance level $\alpha$ is 0.05 . Notation: "**": $p<0.05$ and " $\dagger$ ": the results in the row are from the original papers.

\begin{tabular}{lcccc}
\hline Model & Top-1 acc. & $p$-value & Top-5 acc. & $p$-value \\
\hline ResNet18 (baseline) $\dagger$ & 70.40 & $*$ & 89.45 & $*$ \\
ResNet18+SENet (2018) $\dagger$ & 70.59 & $*$ & 89.78 & $*$ \\
ResNet18+BAM (2018) $\dagger$ & $\underline{71.12}$ & $*$ & $\underline{89.99}$ & $*$ \\
ResNet18+CBAM (2018) $\dagger$ & 70.73 & $*$ & 89.91 & $*$ \\
ResNet18+GPCA (ours) & $\mathbf{7 1 . 6 1} \pm \mathbf{0 . 1 0}$ & N/A & $\mathbf{9 0 . 9 0} \pm \mathbf{0 . 1 3}$ & N/A \\
\hline ResNet50 (baseline) $\dagger$ & 75.20 & $*$ & 92.52 & $*$ \\
ResNet50+SENet (2018) $\dagger$ & 76.71 & $*$ & 93.31 & $*$ \\
ResNet50+BAM (2018) $\dagger$ & 75.98 & $*$ & 92.82 & $*$ \\
ResNet50+CBAM (2018) $\dagger$ & 77.34 & $*$ & 93.66 & $*$ \\
ResNet50+SRP (2019) $\dagger$ & 77.42 & $*$ & 93.79 & $*$ \\
ResNet50+ECA (2020) $\dagger$ & $\underline{77.48}$ & $*$ & 93.68 & $*$ \\
ResNet50+GCT (2020) $\dagger$ & 77.30 & $*$ & $\underline{93.70}$ & $*$ \\
ResNet50+GPCA (ours) & $\mathbf{7 7 . 5 7} \pm \mathbf{0 . 0 7}$ & N/A & $\mathbf{9 3 . 9 7} \pm \mathbf{0 . 0 6}$ & N/A \\
\hline
\end{tabular}

model is larger than $82.5 \%$. At the meantime, the accuracies obtained by the other referred models are all smaller than $82 \%$. It outperforms the base model at about $1.4 \%$ and the best referred method, the SRP with the VGG16 model, at more than $0.8 \%$. Furthermore, the ResNet-based GPCA models also have improvement on classification accuracies on the miniImageNet dataset. Compared with their base models, the GPCA with the ResNet18 and the ResNet34 models increase their accuracies about $1.7 \%$ and $2.1 \%$, respectively, which are considerable performance improvements. Meanwhile, they respectively surpass the CBAM-S with the ResNet18 (78.02\%) and the SRP with the ResNet34 (78.99) models more than $0.8 \%$ and $1.1 \%$, respectively.

Since the $p$-values in Table 3 are all smaller than 0.05, the GPCA achieves statistically significant improvement on classification performance on the minilmageNet dataset.

\subsection{Performance on ImageNet-32 $\times 32$ Dataset}

In Table 4 , the proposed GPCA modules with the VGG16, the ResNet18, and the ResNet34 models perform the best on the ImageNet- $32 \times 32$ dataset and respectively achieve the top- 1 accuracies of $42.58 \%, 49.58 \%$, and $51.24 \%$, and the top- 5 accuracies of $65.65 \%, 75.07 \%$, and $75.79 \%$. Compared with their corresponding base models, the models based on the proposed GPCA module improve the accuracies by a large margin. Applying VGG16 as the base model, the classification accuracy of the GPCA-based model outperforms the second best one, i.e., the SENet module with the VGG16 model, at $2.8 \%$ on top- 1 and $2.7 \%$ on top-5, respectively. Meanwhile, with the ResNet18 base model, the GPCAbased model has considerable performance improvements compared with the base model and the second best model as well. Compared with the base model, the proposed GPCA with the ResNet 18 model achieves $1.8 \%$ and $2.4 \%$ improvements on top- 1 and top- 5 accuracies, respectively. Moreover, it improves the accuracies at $0.7 \%$ on top- 1 and $1.3 \%$ on top5 than the best referred model, i.e., the SRP module with the ResNet18 model. Meanwhile, with the ResNet34 model, the proposed GPCA module achieves the best accuracies on both top- 1 and top- 5 .

Given that the $p$-values in Table 4 are smaller than 0.05 , the GPCA achieves statistically significant performance improvement on the ImageNet- $32 \times 32$ dataset.
TABLE 6: Ablation studies. The means and standard deviations of top-1 test accuracies (\%) on all the datasets. "GPCA w/o prior" means the GPCA with the Gaussian process prior removed. "GPCA-fixed" means the GPCA with fixed parameter $\Theta$. Note that the best results of each base model are marked in bold, respectively.

\begin{tabular}{lcc}
\hline Model & Cifar-10 & Cifar-100 \\
\hline VGG16 (baseline) & $93.90 \pm 0.10$ & $73.64 \pm 0.14$ \\
VGG16+GPCA w/o prior & $94.21 \pm 0.03$ & $73.95 \pm 0.23$ \\
VGG16+GPCA-fixed & $94.50 \pm 0.02$ & $74.59 \pm 0.08$ \\
VGG16+GPCA & $\mathbf{9 4 . 5 1} \pm \mathbf{0 . 0 3}$ & $\mathbf{7 4 . 6 5} \pm \mathbf{0 . 0 1}$ \\
\hline ResNet50 (baseline) & $93.52 \pm 0.05$ & $71.88 \pm 0.06$ \\
ResNet50+GPCA w/o prior & $94.31 \pm 0.13$ & $72.36 \pm 0.20$ \\
ResNet50+GPCA-fixed & $94.87 \pm 0.11$ & $73.84 \pm 0.05$ \\
ResNet50+GPCA & $\mathbf{9 4 . 9 3} \pm \mathbf{0 . 0 1}$ & $\mathbf{7 3 . 9 3} \pm \mathbf{0 . 0 2}$ \\
\hline \hline Model & MiniImageNet & ImageNet-32 $\times 32$ \\
\hline VGG16 (baseline) & $81.36 \pm 0.08$ & $39.70 \pm 0.02$ \\
VGG16+GPCA w/o prior & $81.30 \pm 0.37$ & $41.36 \pm 0.16$ \\
VGG16+GPCA-fixed & $82.52 \pm 0.15$ & $42.15 \pm 0.11$ \\
VGG16+GPCA & $\mathbf{8 2 . 7 8} \pm \mathbf{0 . 0 1}$ & $\mathbf{4 2 . 5 8} \pm \mathbf{0 . 0 1}$ \\
\hline ResNet18 (baseline) & $77.13 \pm 0.06$ & $47.75 \pm 0.07$ \\
ResNet18+GPCA w/o prior & $77.81 \pm 0.24$ & $47.37 \pm 0.13$ \\
ResNet18+GPCA-fixed & $78.85 \pm 0.09$ & $49.34 \pm 0.09$ \\
ResNet18+GPCA & $\mathbf{7 8 . 8 7} \pm \mathbf{0 . 0 2}$ & $\mathbf{4 9 . 5 8} \pm \mathbf{0 . 0 2}$ \\
\hline ResNet34 (baseline) & $78.08 \pm 0.12$ & $49.82 \pm 0.10$ \\
ResNet34+GPCA w/o prior & $78.34 \pm 0.36$ & $50.47 \pm 0.08$ \\
ResNet34+GPCA-fixed & $79.92 \pm 0.06$ & $50.65 \pm 0.05$ \\
ResNet34+GPCA & $\mathbf{8 0 . 1 4} \pm \mathbf{0 . 0 2}$ & $\mathbf{5 1 . 2 4} \pm \mathbf{0 . 0 2}$ \\
\hline
\end{tabular}

\subsection{Performance on ImageNet Dataset}

In Table 5 the proposed GPCA modules with the ResNet18 and the ResNet50 models perform the best on the ImageNet dataset and respectively achieve the top- 1 accuracies of $71.61 \%$ and $77.57 \%$, and the top- 5 accuracies of $90.90 \%$ and $93.97 \%$. Compared with their corresponding base models, the models based on the proposed GPCA module improve the accuracies by a large margin. Moreover, it improves the accuracies at $0.5 \%$ on top- 1 and $1.0 \%$ on top- 5 than the best referred model, i.e., the BAM module with the ResNet18 model. Meanwhile, with the ResNet50 model, the proposed GPCA module achieves the best accuracies on both top1 and top-5. The GPCA achieves statistically significant performance improvement on the ImageNet dataset, as the $p$-values in Table 5 are all smaller than 0.05.

\subsection{Ablation Studies}

Ablation study is conducted and the results are listed in Table 6 To investigate the effectiveness of the optimization, the classification accuracies of the Gaussian processremoved version ("model_name+GPCA wo prior") and the fixed-parameter version ("model_name+GPCA-fixed") are reported. For the Gaussian process-removed version, the means and the variances of each element in $\boldsymbol{U}$ are directly optimized under the backpropagation framework. Then, the attention mask vector $\boldsymbol{V}$ will be generated according to Section 3.1 .

The optimal parameter $\Theta$ of the fixed-parameter version are empirically selected. $\theta_{0}, \theta_{2}$, and $\theta_{3}$ are selected from the set $\{0,0.5,1,2\}$ and they cannot be 0 at the same time. $\theta_{1}$ is selected from the set $\left\{2^{n}\right\}_{n=-2}^{6}$. For different cases (i.e., different base models and datasets), the optimal choices may not be the same. According to our experience, we set $\theta_{1}=1$ 

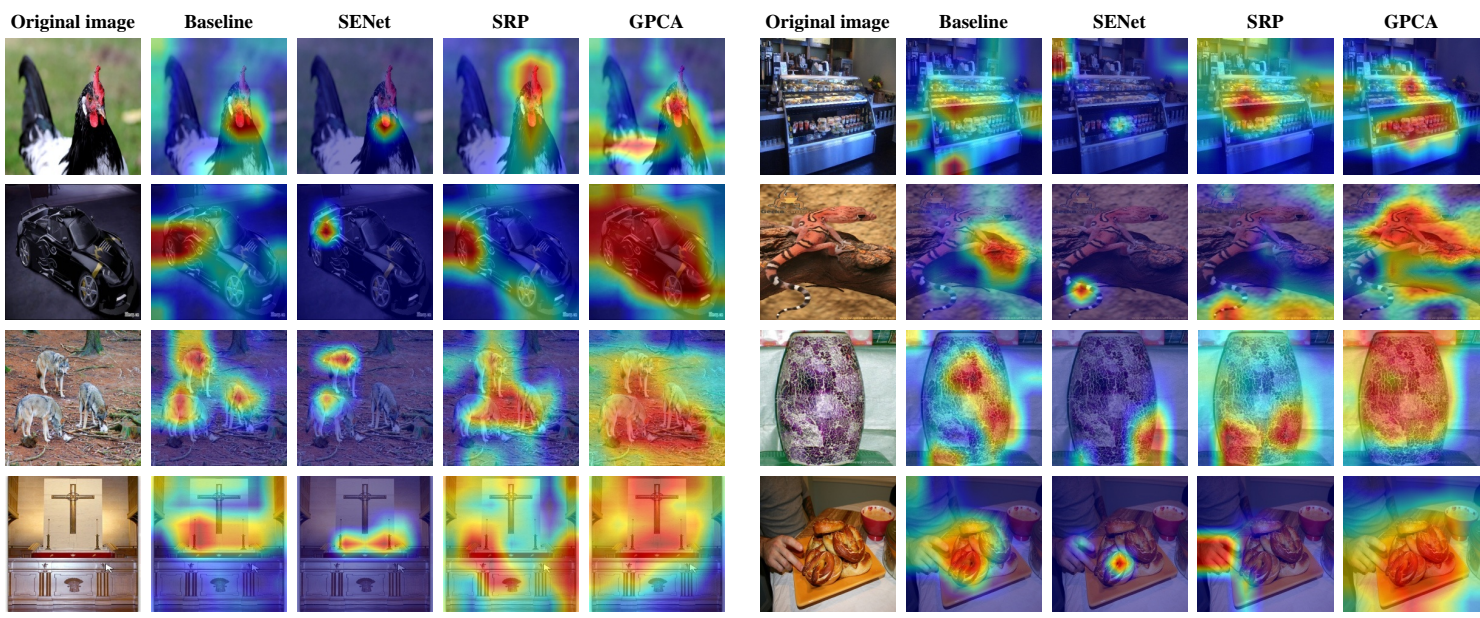

Fig. 5: Visualization of feature maps of the last convolutional layer in the VGG16 model on the miniImageNet dataset. The proposed GPCA module is compared with the baseline (the VGG16 model without any attention modules introduced) and two referred methods (SENet [11] and SRP [19]). Red means the highest concentration, while blue is the fully contrary.

based on the VGG16 model implemented on the Cifar-10 and the Cifar-100 datasets, $\theta_{1}=32$ for the ResNet50 model on the Cifar-10 dataset, $\theta_{1}=0.5$ for the ResNet50 model on the Cifar-100 dataset, and $\theta_{1}=64$ for all the other cases. In all these cases, $\theta_{0}=1, \theta_{2}=0$, and $\theta_{3}=1$ are fixed. In summary, $\theta_{1}$ is sensitive for GP training in different cases, while the other three parameters are less sensitive. Other implementation details are the same as those in Section 4.2

We list means and standard deviations of the classification accuracy, and the $p$-values of the Student's $t$-test in Table 6 The proposed GPCA achieves statistically significantly better performance than the corresponding base models on all the datasets. Meanwhile, the mean performance of the GPCA is better than that of both "GPCA w/o prior" and "GPCA-fixed" cases, with smaller standard deviations. This indicates that the Gaussian process prior and the parameter optimization are the essential parts of the GPCA module.

\subsection{Visualization}

Visualization of the proposed GPCA is shown in Figure 5 We compare the proposed GPCA method with the baseline (VGG16 without any attention modules) and two referred methods on the miniImageNet dataset. The compared methods include SENet [11] (a popular channel attention method) and SRP [19] (it achieves the best performance among all the referred methods). We applied the gradient-weighted class activation mapping (GradCAM) [60] method to implement the visualization. Eight images and their corresponding visualization are illustrated.

As shown in Figure 5, the GPCA method focuses on larger discriminative parts of the objects in the images. The key regions (in red) usually enclose the whole object to benefit classification. At the meantime, the other methods only concentrate on relatively small region in the images.

For example, in the second sample of the left column, a car is in the image. The baseline, the SENet, and the SRP can find parts of the car, while the GPCA can detect the car as a whole. Another example, the fourth sample of the right column, shows multiple pretzels (targets) on the table and in a hand. The baseline and the SENet can find parts of the pretzels, but the SRP merely focuses on the hand. At the meantime, the GPCA concentrates on all the pretzels. These indicate that the GPCA has ability to find more informative channels than the others such that we can integrate them for better classification performance.

\section{Experiments on Weakly Supervised Ob- JECT LOCALIZATION}

In this section, we evaluate the performance of the proposed GPCA module on one benchmark dataset for the weakly supervised object localization (WSOL) task. We compare the proposed GPCA module with two attention modules, i.e., SENet [11], and SRP [19], and state-of-theart WSOL methods including class activation mapping (CAM) [61], attention-based dropout layer (ADL) [50], [51], adversarial complementary learning (ACoL) |62], hide-andseek $(\mathrm{HaS})[63]$, self-produced guidance (SPG) [64], divergent activation network (DANet) [65], and dual-attention guided dropblock module (DGDM) |66|.

\subsection{Dataset}

CUB-200-2011 [67] dataset is used for the WSOL task. The CUB-200-2011 dataset contains 200 categories of birds with 5,994 images for training and 5,794 images for test. It is mainly motivated for fine-grained visual classification (FGVC) tasks, while previous researches [50], [51], [61], [62], [63], 64], [65] commonly worked on it for WSOL.

\subsection{Implementation Details}

VGG16, MobileNet [68], and ResNet50 models were used as the base models. Adopting the SGD optimizer, we trained each model 100 epochs with batch size of 32 , and the initial learning rates were set as 0.01 and decayed by a factor of 10 at the $80^{\text {th }}$ epoch. The momentum and the weight decay values were kept as 0.9 and $5 \times 10^{-4}$, respectively. All the models with the attention modules were fine-tuned on ImageNet-pretrained models and conducted three times with different random initializations. The means and the standard deviations of the localization and the classification accuracies are reported, respectively. A correct localization means that the network can predict a correct class and the intersection over union (IoU) between the estimated box and the ground truth is no smaller than $50 \%$. The localization accuracy can present the performance of the network more accurate than the classification accuracy. 
TABLE 7: The means and standard deviations of top-1 and top-5 localization accuracies (loc. acc., \%), the classification accuracies (cls. acc., \%), and the $p$-values ( $p$ ) of the Student's $t$-test between the accuracies of the proposed method and the other methods on the CUB-200-2011 dataset. Note that the best results of each base model are marked in bold, respectively. We set the significance level $\alpha$ as 0.05 . Notation: "-": no results, "*": $p<0.05$. $\dagger$ means the results in the row are from the original papers.

\begin{tabular}{|c|c|c|c|c|c|c|c|c|c|}
\hline \multirow[b]{2}{*}{ Base model } & \multirow[b]{2}{*}{ Method } & \multicolumn{4}{|c|}{ Loc. acc. } & \multicolumn{4}{|c|}{ Cls. acc. } \\
\hline & & Top-1 acc. & $p$-value & Top-5 acc. & $p$-value & Top-1 acc. & $p$-value & Top-5 acc. & $p$-value \\
\hline \multirow{18}{*}{ VGG16 } & $\operatorname{ADL}(2019)^{\dagger}$ & 52.36 & * & - & - & 65.27 & * & - & - \\
\hline & $\operatorname{ACoL}(2018)^{\dagger}$ & 45.92 & * & 56.51 & * & 71.9 & * & - & - \\
\hline & SPG $(2018)^{\dagger}$ & 48.93 & * & 57.85 & * & 75.5 & & 92.1 & \\
\hline & CAM (2016) & $42.15 \pm 0.88$ & * & $51.04 \pm 0.92$ & * & $75.38 \pm 0.20$ & & $92.26 \pm 0.36$ & \\
\hline & ADL (2019) & $45.30 \pm 0.26$ & * & $54.57 \pm 0.26$ & * & $73.18 \pm 0.50$ & $*$ & $91.97 \pm 0.37$ & * \\
\hline & ADL-SE (2018) & $52.62 \pm 0.38$ & * & $68.27 \pm 0.29$ & * & $73.54 \pm 0.18$ & * & $91.42 \pm 0.31$ & * \\
\hline & ADL-SRP (2019) & $52.07 \pm 0.59$ & * & $68.11 \pm 0.58$ & * & $74.26 \pm 0.34$ & * & $90.68 \pm 0.35$ & * \\
\hline & ADL-GPCA (ours) & $54.97 \pm 0.35$ & $\mathrm{~N} / \mathrm{A}$ & $69.68 \pm 0.24$ & $\mathrm{~N} / \mathrm{A}$ & $75.62 \pm 0.17$ & $\mathrm{~N} / \mathrm{A}$ & $92.77 \pm 0.32$ & $\mathrm{~N} / \mathrm{A}$ \\
\hline & DANet $(2019)^{\dagger}$ & 52.52 & & 61.96 & & 75.40 & & 92.30 & \\
\hline & DANet (2019) & $50.96 \pm 0.52$ & * & $60.85 \pm 0.50$ & * & $74.81 \pm 0.42$ & & $92.15 \pm 0.18$ & \\
\hline & DANet-SE (2018) & $51.19 \pm 0.23$ & * & $61.00 \pm 0.06$ & * & $74.83 \pm 0.36$ & & $92.22 \pm 0.30$ & \\
\hline & DANet-SRP (2019) & $50.70 \pm 0.53$ & * & $60.96 \pm 0.47$ & * & $74.05 \pm 0.40$ & * & $92.07 \pm 0.12$ & \\
\hline & DANet-GPCA (ours) & $52.59 \pm 0.38$ & $\mathrm{~N} / \mathrm{A}$ & $62.05 \pm 0.18$ & $\mathrm{~N} / \mathrm{A}$ & $74.77 \pm 0.39$ & $\mathrm{~N} / \mathrm{A}$ & $92.36 \pm 0.32$ & $\mathrm{~N} / \mathrm{A}$ \\
\hline & DGDM $(2020)^{\dagger}$ & 54.34 & * & - & - & 69.85 & & - & - \\
\hline & DGDM (2020) & $52.80 \pm 0.53$ & * & $67.82 \pm 0.39$ & * & $69.34 \pm 0.16$ & * & $90.26 \pm 0.06$ & * \\
\hline & DGDM-SE (2018) & $53.17 \pm 0.44$ & * & $67.55 \pm 0.11$ & * & $69.29 \pm 0.33$ & * & $90.11 \pm 0.32$ & * \\
\hline & DGDM-SRP (2019) & $53.65 \pm 0.38$ & * & $68.27 \pm 0.72$ & * & $69.03 \pm 0.31$ & * & $90.13 \pm 0.22$ & * \\
\hline & DGDM-GPCA (ours) & $54.90 \pm 0.18$ & $\mathrm{~N} / \mathrm{A}$ & $69.80 \pm 0.09$ & $\mathrm{~N} / \mathrm{A}$ & $69.67 \pm 0.26$ & $\mathrm{~N} / \mathrm{A}$ & $90.90 \pm 0.31$ & N/A \\
\hline \multirow{7}{*}{ MobileNet } & HaS-32 $(2017)^{\dagger}$ & 44.67 & * & - & - & 66.64 & * & - & - \\
\hline & $\operatorname{ADL}(2019)^{\dagger}$ & 47.74 & * & - & - & 70.43 & * & - & - \\
\hline & CAM (2016) & $42.81 \pm 0.07$ & * & $53.24 \pm 0.76$ & * & $69.17 \pm 0.86$ & * & $90.11 \pm 0.25$ & * \\
\hline & ADL (2019) & $45.09 \pm 0.23$ & * & $54.32 \pm 0.40$ & * & $72.93 \pm 0.24$ & * & $90.96 \pm 0.33$ & * \\
\hline & ADL-SE (2018) & $48.67 \pm 0.37$ & * & $59.07 \pm 0.41$ & * & $73.05 \pm 0.36$ & * & $91.65 \pm 0.36$ & \\
\hline & ADL-SRP (2019) & $48.15 \pm 0.78$ & * & $58.61 \pm 0.58$ & * & $71.98 \pm 0.20$ & * & $90.08 \pm 0.38$ & * \\
\hline & ADL-GPCA (ours) & $50.51 \pm 0.40$ & $\mathrm{~N} / \mathrm{A}$ & $61.28 \pm 0.23$ & $\mathrm{~N} / \mathrm{A}$ & $73.92 \pm 0.25$ & $\mathrm{~N} / \mathrm{A}$ & $91.97 \pm 0.12$ & $\mathrm{~N} / \mathrm{A}$ \\
\hline \multirow{10}{*}{ ResNet50 } & CAM (2016) & $42.82 \pm 0.74$ & * & $52.13 \pm 0.82$ & * & $74.68 \pm 0.77$ & * & $92.89 \pm 0.18$ & * \\
\hline & ADL (2019) & $45.74 \pm 0.10$ & * & $54.45 \pm 0.14$ & * & $76.86 \pm 0.20$ & * & $91.26 \pm 0.06$ & * \\
\hline & ADL-SE (2018) & $49.03 \pm 0.17$ & * & $58.72 \pm 0.23$ & & $77.86 \pm 0.26$ & * & $93.95 \pm 0.07$ & \\
\hline & ADL-SRP (2019) & $45.55 \pm 0.25$ & * & $54.62 \pm 0.08$ & * & $76.06 \pm 0.05$ & * & $91.83 \pm 0.07$ & * \\
\hline & ADL-GPCA (ours) & $50.73 \pm 0.47$ & $\mathrm{~N} / \mathrm{A}$ & $59.66 \pm 0.63$ & $\mathrm{~N} / \mathrm{A}$ & $78.75 \pm 0.08$ & $\mathrm{~N} / \mathrm{A}$ & $94.17 \pm 0.28$ & N/A \\
\hline & DGDM $(2020)^{\dagger}$ & 59.40 & * & - & - & 76.20 & & - & - \\
\hline & DGDM (2020) & $57.87 \pm 0.34$ & * & $69.71 \pm 0.33$ & * & $76.23 \pm 0.23$ & & $92.64 \pm 0.19$ & \\
\hline & DGDM-SE (2018) & $62.25 \pm 0.43$ & * & $76.29 \pm 0.20$ & * & $76.37 \pm 0.19$ & * & $92.77 \pm 0.07$ & \\
\hline & DGDM-SRP (2019) & $62.33 \pm 0.24$ & * & $76.38 \pm 0.36$ & $*$ & $75.84 \pm 0.34$ & & $92.63 \pm 0.22$ & \\
\hline & DGDM-GPCA (ours) & $63.22 \pm 0.27$ & $\mathrm{~N} / \mathrm{A}$ & $77.20 \pm 0.18$ & $\mathrm{~N} / \mathrm{A}$ & $75.80 \pm 0.23$ & $\mathrm{~N} / \mathrm{A}$ & $92.73 \pm 0.20$ & $\mathrm{~N} / \mathrm{A}$ \\
\hline
\end{tabular}

In addition, the two-sample Student's $t$-tests have been conducted between the accuracies of the proposed method and the other referred methods that we reimplemented. As the work in WSOL always reports results for one run, we conducted the one-sample Student's $t$-tests between the accuracies of the proposed method and the WSOL methods in these work. We set the significance level $\alpha$ as 0.05 .

For the ADL- and DGDM-based models, we applied ADL or DGDM after each pooling and strided convolutional layer. In addition, for all the models, we replaced the last pooling layer and the FC layer(s) with two $3 \times 3$ convolutional layers, one $1 \times 1$ convolutional layer for mapping output the channel index to the class index, and one GAP for shrinking channels to scalars, following [65]. All the attention modules were only applied before the GAP(s).

\subsection{Performance on CUB-200-2011 Dataset}

Experimental results in the WSOL task are reported in Table 7. It can be observed that the proposed GPCA module performs better on both top- 1 and top- 5 localization accuracies than the SENet and the SRP in all the three base models with three different WSOL methods as baselines, respectively, and achieves statistically significant improvement on localization accuracies, instead of the top- 5 accuracies of the SENet with the ADL in the ResNet50 model. Meanwhile, compared with the baseline models, the proposed GPCA module can obtain statistically significant improvement on localization as well. The GPCA module performs better than the referred methods, which indicates that the GPCA module can effectively improve feature representation ability.

In addition, we visualize a group of localization results in images from the test set of the CUB-200-2011 dataset (Figure 6). From the Figure 6, the proposed GPCA modulebased model with the ADL can locate more precise object regions such that the localization performance is improved.

\section{EXPERIMENTS ON OBJect Detection}

In this section, we evaluate the performance of the GPCA on two benchmark datasets for object detection task. We compare the proposed GPCA module with four attention modules, i.e., SENet [11], SRP [19], ECA [40], and GCT [41], as all the attention modules can be applied in the detectors.

\subsection{Dataset}

We utilize the PASCAL visual object classes challenge 2007 (VOC2007) [69| and common objects in context (COCO) |70| datasets, which are well-known and widely used benchmark datasets, for evaluations of the object detection task. The VOC2007 dataset contains 20 classes with a training set (5, 011 images) and a test set (4,952 images). The COCO dataset contains 80 classes with a training set $(118,287$ images) and a test set (40,670 images). 

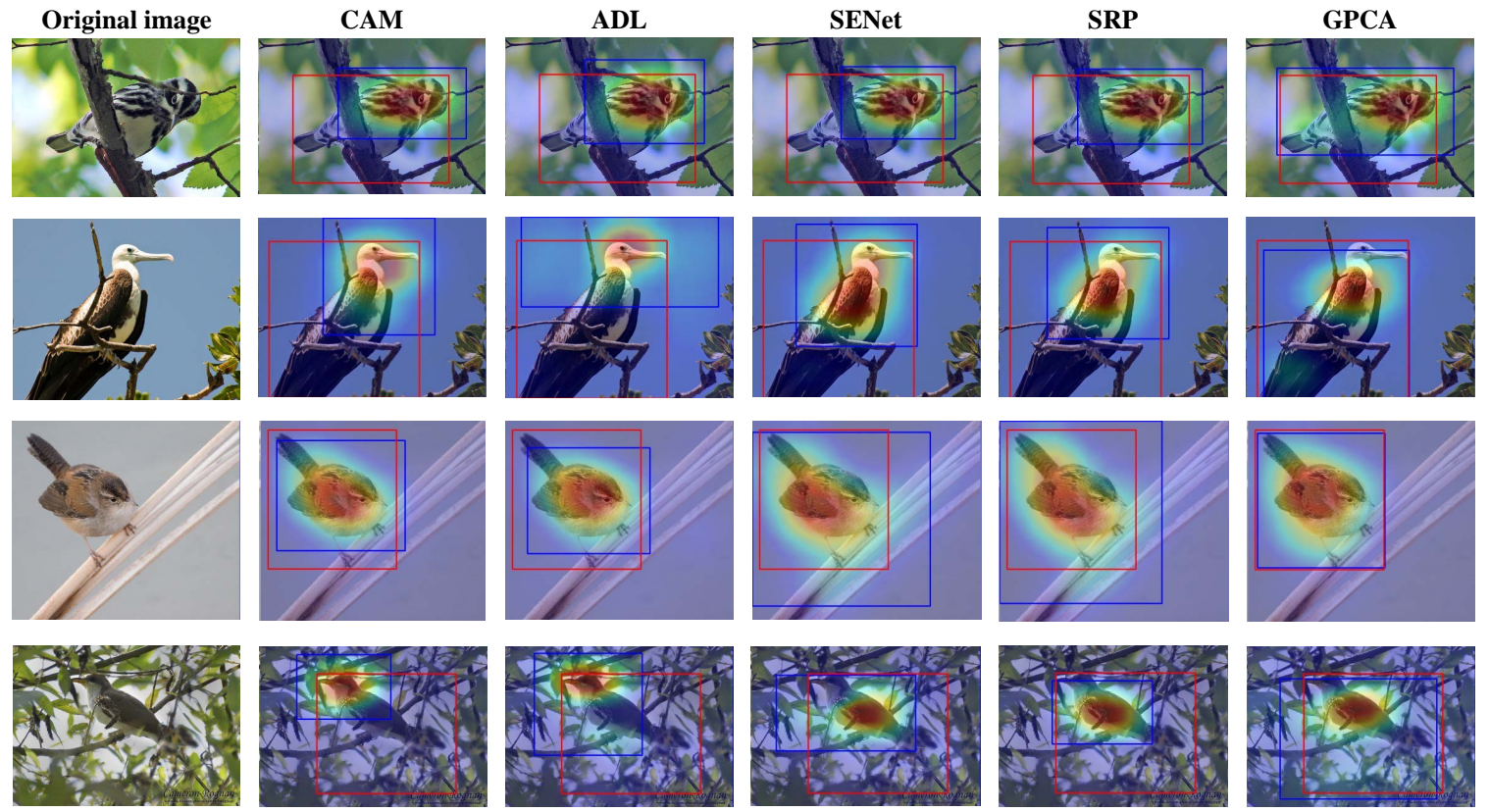

Fig. 6: Examples of WSOL results on the CUB-200-2011 dataset. The proposed GPCA module is compared with two attention modules (SENet and SRP) and two WSOL methods (CAM and ADL). The correctly predicted WSOL results are shown. The blue bounding boxes are the predicted results and the red ones are the ground truths.

\subsection{Implementation Details}

On the VOC2007 dataset, single shot detector (SSD) [5] model, which applies the VGG16 model as its backbone, was used as the base model for the attention modules. Meanwhile, all the attention modules were only applied after the Conv5_3 of the VGG16 model for enhancing feature representation. Two settings, (1) training on the VOC2007 and testing on it with an ImageNet-pretrained backbone and $300 \times 300$ input image sizes, (2) training on the VOC2007 and VOC2012 and testing on the VOC2007 with a COCO-pretrained backbone and $512 \times 512$ input image sizes, were undertaken. For the training process, we followed the original strategy in [5]. All the models with the attention modules were conducted three times with random initialization. The means of average precision (AP, \%) of each class, and the means and the standard deviations of the mean average precision ( $\mathrm{mAP}, \%)$ of all the classes are reported, respectively. The intersection over union (IoU) for calculating the AP is set as $50 \%$.

On the COCO dataset, fully convolutional one-stage object detector (FCOS) [71] and Adaptive Training Sample Selection (ATSS) [72] were applied as the base models for the attention modules, respectively. ResNet50 was used as the backbone of the two detectors. All the attention modules were cascaded after each outputs of the feature pyramid network (FPN) [73]. Adopting the SGD optimizer, we trained each model twelve epochs with batch size of eight, and the initial learning rates were set as 0.01 and decayed twice by a factor of 10 at the eighth and eleventh epochs, respectively. The momentum and the weight decay values were kept as 0.9 and $5 \times 10^{-4}$, respectively. Six evaluation metrics were considered including averaged APs for small, medium, and large scale objects and overall at $[50 \%, 95 \%]$ IoU interval with step as $5 \%$, and APs at IoU as $50 \%$ and $75 \%$. We conducted the experiments with MMDetection |74|.

In addition, the two-sample Student's $t$-tests have been conducted between the mAP of the proposed method and the other referred methods that we reimplemented.

\subsection{Performance on VOC2007 Dataset}

The experimental results of the VOC2007 dataset are shown in Table 8. When training on VOC2007 only, the proposed GPCA module achieves statistically significant improvement on mAP at $70.49 \%$ on average and outperforms the second best one, i.e., the GCT module $(69.75 \%)$, by $0.75 \%$. For all the 20 classes, the GPCA module performs best on nine classes and the second best on the other eight classes. For the other three classes, the GPCA module obtains small gaps compared with corresponding best attention modules, and is ranked third, respectively. When training on both VOC2007 and VOC2012 with larger input image sizes, the proposed GPCA module can further obtain $\mathrm{mAP}$ at $81.92 \%$ on average, which also performs statistically significant improvement among the referred methods. Meanwhile, the GPCA module performs best on eleven classes and the second best on the other four classes. These indicate the significant performance of the GPCA module.

We also illustrate four detection examples which are randomly selected from the test set of the VOC2007 dataset in Figure 7. For each attention module-based model and the baseline model, the predicted bounding boxes of each class are drawn with different colors. From the Figure 7 the proposed GPCA module-based model finds all the objects in the images with high IoU between the predictions and the corresponding ground truth. Although the compared methods can perform well sometimes, they merely detect a part of the objects and predict with low IoU in some cases.

\subsection{Performance on COCO Dataset}

The experimental results of the COCO dataset are shown in Table 9 According to Table 9 the proposed GPCA module can outperform the referred attention modules with the two base models, respectively, on the six evaluation metrics. On 
TABLE 8: The means of AP (\%) of each class, the means and standard deviations of mAP (\%) on the VOC2007 dataset, and the $p$-values $(p)$ of the Student's $t$-test between the mAPs of the proposed method and the other methods. Note that the best and second best results are marked in bold and italic, respectively. We set the significance level $\alpha$ as 0.05 . Notation: "*": $p<0.05$. The class "Mbike" means motorbike.

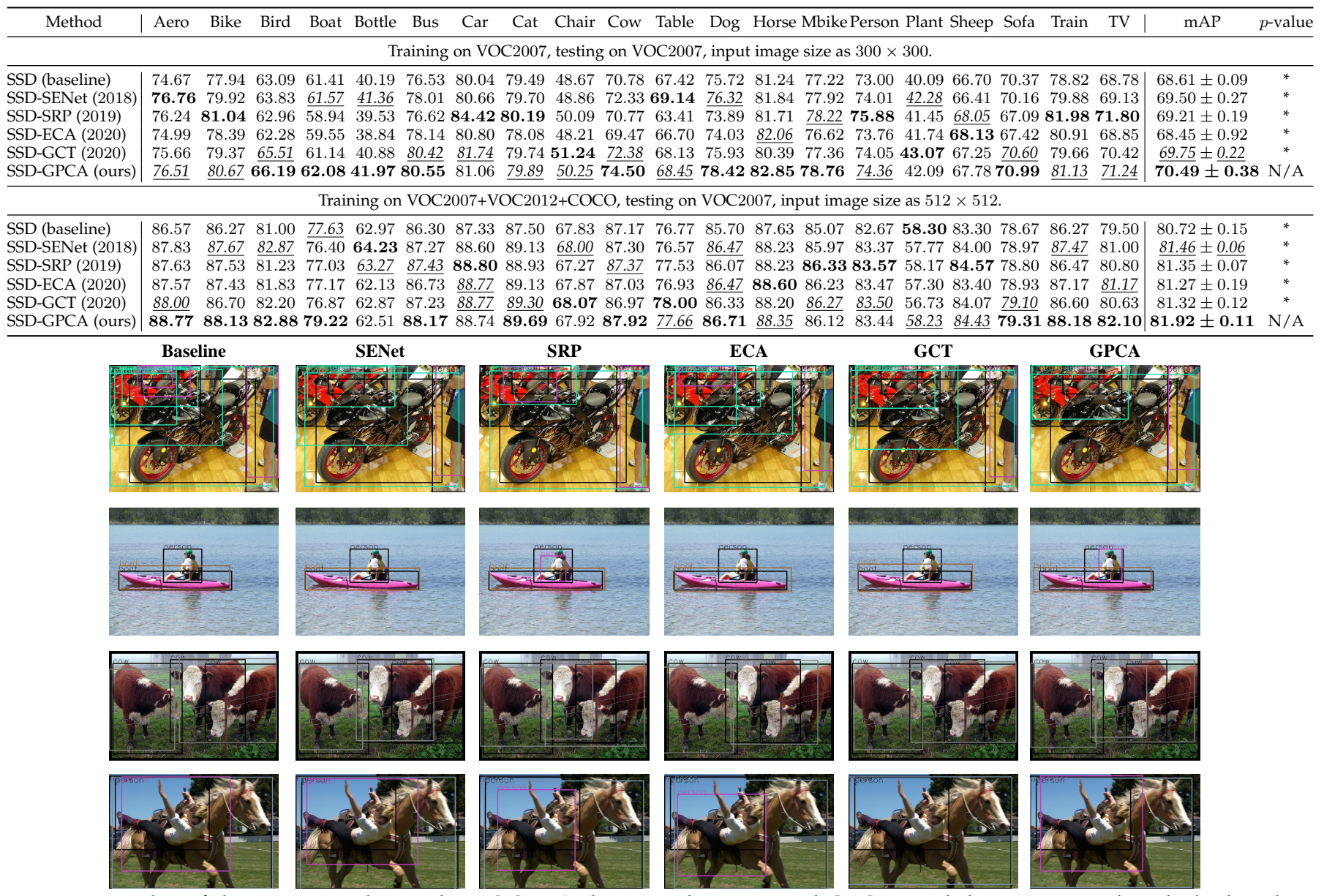

Fig. 7: Examples of detection results on the VOC2007 dataset. The proposed GPCA module is compared with the baseline (the SSD model without any attention modules introduced) and four referred methods (SENet, SRP, ECA, and GCT). The correct predicted detection results with IoU higher than 0.5 are shown. Each color corresponds to an object category (green: motor bike; pink: person; brown: boat; grey: cow; light blue: horse) and the black bounding boxes are the ground truths.

the prime challenge metric, i.e., the AP metric, the GPCA module can achieve $38.50 \%$ with the FCOS and $39.20 \%$ with the ATSS. Moreover, on most of the evaluation metrics, the proposed GPCA module performs statistically significant improvement compared with the referred methods, except the $\mathrm{AP}_{50}$ with the FCOS and the $\mathrm{AP}_{\mathrm{L}}$ with the ATSS. These can also demonstrate the significant effectiveness of the GPCA module.

\section{EXPERIMENTS ON SEMANTIC SEgmENTATION}

In this section, we evaluate the performance of GPCA for the task of semantic segmentation. We compare the proposed GPCA module with SENet [11] and ECA [40].

\subsection{Dataset}

We utilize the CityScapes [76] dataset for evaluations. It contains 19 classes with 3,475 annotated images for training and validating, and 1,525 images for test.

\subsection{Implementation Details}

On the CityScapes dataset, the object-contextual representation network (OCRNet) [7] model, which applies HRNetV2W18 and HRNetV2-W48 [77| models as its backbones, was used as the base model for the attention modules. Meanwhile, all the attention modules were only applied after the OCR module for enhancing feature representation. During the training process, we followed the original strategy in MMSegmentation |75| with 80,000 iterations and batch size of 4 due to limited GPU resources ( 8 for the original setting). The two backbones with all the attention modules were conducted three times with random initialization, respectively. The means and the standard deviations of the mean intersection over union (mIoU, \%) and mean accuracy (mACC, \%) are reported.

\subsection{Performance on CityScapes Dataset}

The experimental results of the CityScapes dataset are listed in Table 10 According to Table 10, the proposed GPCA module can achieve $80.09 \%$ and $87.01 \%$ for mIoU and $\mathrm{mAcc}$ with HR18, respectively. With HR48, the mIoU and mACC are $80.65 \%$ and $88.07 \%$, respectively. All these values are larger than those obtained by the referred methods. The $p$ values of the student's- $t$ test between the proposed GPCA module and the reimplemented indicate the statistically significant improvement on segmentation performance. 
TABLE 9: Means and standard deviations of AP (\%) in different cases on the COCO dataset, and the $p$-values $(p)$ of the Student's $t$-test between the APs of the proposed method and the other methods. Note that the best and second best results are marked in bold and italic, respectively. We set the significance level $\alpha$ as 0.05 . Notation: "**": $p<0.05$, "AP", "AP", " $\mathrm{AP}_{\mathrm{M}}$ ", and " $\mathrm{AP}_{\mathrm{L}}$ ": averaged $\mathrm{AP}$ for overall, small, medium, and large scale objects, respectively, at [50\%, $\left.95 \%\right]$ IoU interval with step as $5 \%$, "AP $\mathrm{A}_{50}$ " and "AP 75 ": $\mathrm{AP}$ at IoU as $50 \%$ and $75 \%$, respectively.

\begin{tabular}{|c|c|c|c|c|c|c|c|c|c|c|c|c|}
\hline Method & $\mathrm{AP}$ & $p$-value & $\mathrm{AP}_{50}$ & $p$-value & $\mathrm{AP}_{75}$ & $p$-value & $\mathrm{AP}_{\mathrm{S}}$ & $p$-value & $\mathrm{AP}_{\mathrm{M}}$ & $p$-value & $\mathrm{AP}_{\mathrm{L}}$ & $p$-value \\
\hline FCOS (ba & $37.50 \pm 0.10$ & 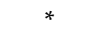 & $56.43 \pm 0.21$ & & $40.27 \pm 0.15$ & * & $21.50 \pm 0.30$ & & $41.03 \pm 0.25$ & * & $48.60 \pm 0.26$ & . \\
\hline FCOS-SE (2018) & $37.50 \pm 0.22$ & * & $55.90 \pm 0.16$ & * & $40.78 \pm 0.26$ & * & $21.40 \pm 0.33$ & * & $41.53 \pm 0.13$ & * & $48.48 \pm 0.38$ & 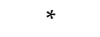 \\
\hline FCOS-ECA (2020) & $\underline{37.90} \pm \underline{0.10}$ & * & $\underline{56.77} \pm \underline{0.67}$ & & $\underline{41.07} \pm \underline{0.12}$ & & $\underline{21.63} \pm \underline{0.40}$ & * & $\underline{41.67} \pm \underline{0.12}$ & $*$ & $\underline{48.67} \pm \underline{0.15}$ & * \\
\hline FCOS-GPCA (ours) & $38.50 \pm 0.20$ & $\mathrm{~N} / \mathrm{A}$ & $5 \overline{6.80} \pm \overline{0.35}$ & $\mathrm{~N} / \mathrm{A}$ & $41.50 \pm \overline{0.35}$ & $5 \mathrm{~N} / \mathrm{A}$ & $22.17 \pm 0.29$ & $9 \mathrm{~N} / \mathrm{A}$ & $4 \overline{2.23} \pm \overline{0.15}$ & $\mathrm{~N} / \mathrm{A}$ & $49.73 \pm \overline{0.55}$ & $5 \mathrm{~N} / \mathrm{A}$ \\
\hline ATSS (base & $.75 \pm$ & $*$ & $56.35 \pm 0.07$ & * & $41.80 \pm 0.14$ & * & $22.55=$ & 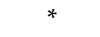 & $42.20 \pm$ & * & 48.95 & * \\
\hline ATSS-SE (2018) & $38.95 \pm 0.07$ & * & $\underline{57.15} \pm \underline{0.07}$ & 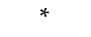 & $\underline{42.00} \pm \underline{0.03}$ & * & $\underline{23.00} \pm \underline{0.14}$ & * & $\underline{42.25} \pm \underline{0.07}$ & * & $\underline{50.15} \pm \underline{0.07}$ & \\
\hline ATSS-ECA (2020) & $\underline{39.00} \pm \underline{0.01}$ & * & $\overline{56.95} \pm \overline{0.07}$ & * & $\overline{41.95} \pm \overline{0.07}$ & * & $\overline{22.75} \pm \overline{0.07}$ & * & $\overline{41.90} \pm \overline{0.14}$ & * & $\overline{50.05} \pm \overline{0.07}$ & \\
\hline ATSS-GPCA (ours) & $\overline{39.20} \pm \overline{0.01}$ & $\mathrm{~N} / \mathrm{A}$ & $57.55 \pm 0.07$ & $\mathrm{~N} / \mathrm{A}$ & $42.45 \pm 0.07$ & $7 \mathrm{~N} / \mathrm{A}$ & $23.55 \pm 0.07$ & $7 \mathrm{~N} / \mathrm{A}$ & $42.70 \pm 0.02$ & $2 \mathrm{~N} / \mathrm{A}$ & $50.65 \pm 0.35$ & $5 \mathrm{~N} / \mathrm{A}$ \\
\hline
\end{tabular}

TABLE 10: The means and standard deviations of mIoU $(\%)$ and $\mathrm{mACC}(\%)$ for the task of semantic segmentation in different backbones on the CityScapes dataset, the $p$ values $(p)$ of the Student's $t$-test between mIoUs or mACCs of the proposed method and the other methods. Note that the best and second best results are marked in bold and italic, respectively. We set the significance level $\alpha$ as 0.05 . HR18 and HR48 means HRNetV2-W18 and HRNetV2-W48, respectively. " "*" means statistically significant difference. " $\dagger$ " means the results in the row are from [75] with 80,000 iterations and batch size of 8 .

\begin{tabular}{|c|c|c|c|c|c|}
\hline Method & Backbone & $\mathrm{mIoU}$ & $p$-value & $\mathrm{mAcc}$ & $p$-value \\
\hline OCR (baseline) ${ }^{\dagger}$ & & 78.56 & * & 86.04 & * \\
\hline OCR (baseline) & & $78.92 \pm 0.14$ & * & $86.45 \pm 0.13$ & * \\
\hline OCR-SE (2018) & HR18 & $79.51 \pm 0.25$ & * & $86.72 \pm 0.14$ & * \\
\hline OCR-ECA (2020) & & $\overline{79.47} \pm \overline{0.29}$ & * & $\overline{86.46} \pm \overline{0.24}$ & * \\
\hline OCR-GPCA (ours) & & $80.09 \pm 0.22$ & $2 \mathrm{~N} / \mathrm{A}$ & $87.01 \pm 0.20$ & $\mathrm{~N} / \mathrm{A}$ \\
\hline 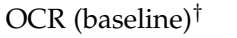 & & 80.70 & & 88.11 & \\
\hline OCR (baseline) & & $80.18 \pm 0.12$ & * & $87.36 \pm 0.30$ & * \\
\hline OCR-SE (2018) & HR48 & $80.32 \pm 0.20$ & * & $87.66 \pm 0.14$ & * \\
\hline OCR-ECA (2020) & & $80.35 \pm 0.15$ & * & $\overline{87.60} \pm \overline{0.26}$ & * \\
\hline OCR-GPCA (ours) & & $8 \overline{80.65} \pm \overline{0.13}$ & $3 \mathrm{~N} / \mathrm{A}$ & $88.07 \pm 0.32$ & $\mathrm{~N} / \mathrm{A}$ \\
\hline
\end{tabular}

It is worth to note that our mean mIoU and mAcc with the HRNetV2-W48 are both slightly lower than the ones reported in [75], which may be caused by the smaller batch size in our implementations. However, there is no statistically significant difference between them. Furthermore, we find the highest mIoU and mAcc of ours out of three times of simulations are $80.81 \%$ and $88.43 \%$, respectively. These results are higher than the officially reported ones. This indicates that, although the proposed GPCA module was affected by the smaller batch size setting, it still has competitive performance compared with the official results.

\section{Analysis of the GPCA Module}

\subsection{The Role of the GPCA Module}

To investigate the role of the GPCA in CNNs, we illustrate the distribution of the values of the attention masks learned by the SENet and the proposed GPCA module for different classes. We selected five classes, i.e., goldfish, spoonbill, airship, pinwheel, and washbasin, from the ImageNet$32 \times 32$ dataset and illustrate the averaged values of the attention masks on each channel of all the test samples and the histograms of the values in the corresponding classes in Figure 8 ResNet18 has been selected as the base model. The modules in each subfigure are named as GPCA_stageID_blockID. Note that the same channels between the SENet and the proposed GPCA do not always indicate same features, which means we cannot directly compare the values of their attention masks channel by channel. In addition to the averaged values of the attention masks, we select two blocks, i.e., GPCA_2_1 and GPCA_3_1, to show their boxplots in Figure 9 For better illustration, eight channels of the aforementioned blocks are selected at equal intervals.

It can be observed that, in most of the channels, the masks of GPCA for different classes are more distinct than those of SENet. For different channels, the GPCA masks vary dramatically along the channel index. However, for more than half of the channels, the SENet masks are similar in values. These observations suggest that the proposed GPCA module has better ability in learning the channel information than the SENet. This is because different values of the attention masks (i.e., importance weights) are assigned to each channel by GPCA and show their distinct roles, while the SENet tends to assign the channels equal weights and, therefore, the attention scheme does not work well.

Thus, the effectiveness of the GPCA module can be clarified since the GPCA modules perform as channel-wise feature selectors and play distinct and important roles in different depths of the ResNet18 model. In addition, similar phenomena can be also observed on the other datasets, and in the VGG16 and the ResNet34 models, respectively. We should note that the attention masks, which perform as weights of channels, reflect the importance of the corresponding channels, rather than directly demonstrating the correlations between the channels, although the attention masks are estimated according to the channel correlations.

\subsection{Ability of Learning the Channel Correlations}

In this section, we intuitively evaluate the attention modules' abilities in learning the channel correlations to clarify the actual benefits and advantages of the proposed GPCA module. Given that the final FC layers are replaced by convolutional layers following a global average pooling (GAP) and the output number of channels of the last convolutional layer is aligned to the number of classes in the WSOL task (see Section 5.2), the attention modules can be employed before the GAP and play a role in selecting a true channel (if possible) for the prediction by setting higher attention masks for the ground truth classes without supervision. In this case, the classification labels can be treated as the pseudo targets of the attention modules and evaluate the attention modules explicitly. 


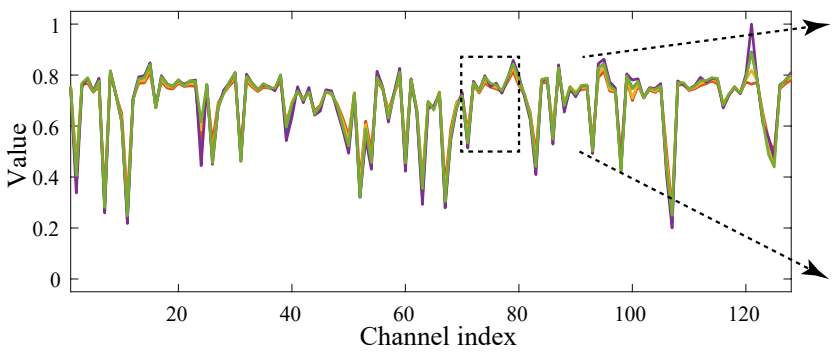

(a) Attention mask values of SENet_3_1

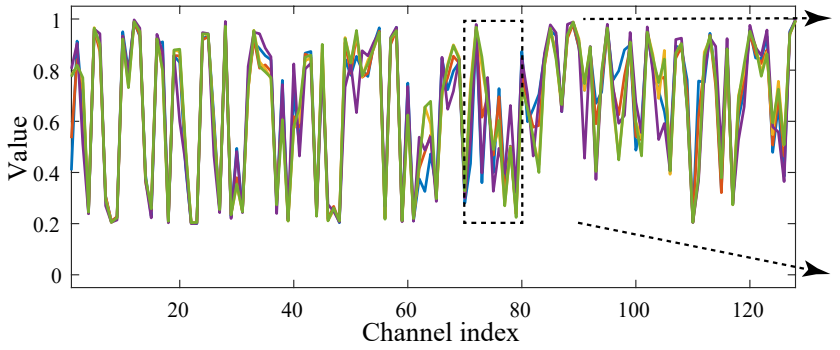

(d) Attention mask values of GPCA_3_1

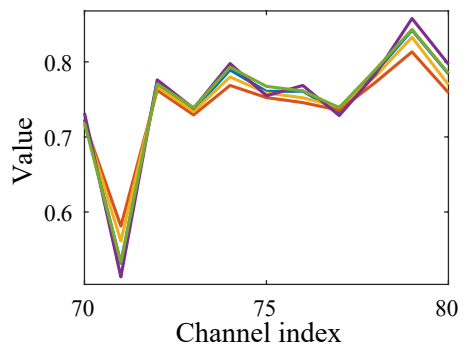

(b) Channel 70-80 of SENet 3

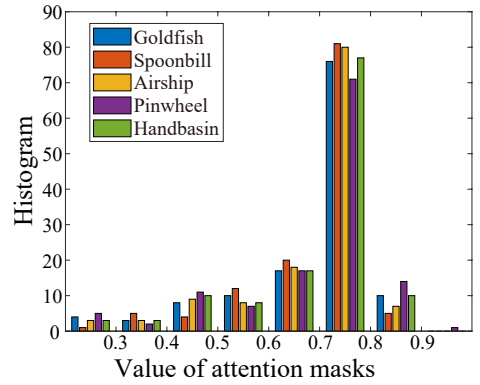

(c) Histogram of SENet_3_1

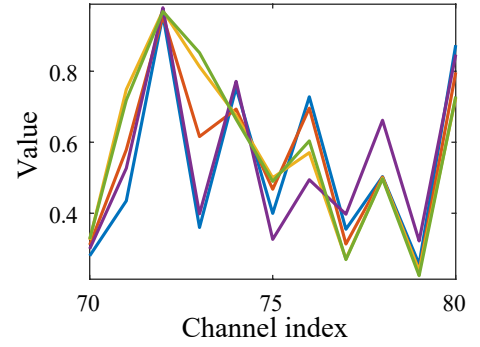

(e) Channel 70-80 of GPCA_3_1

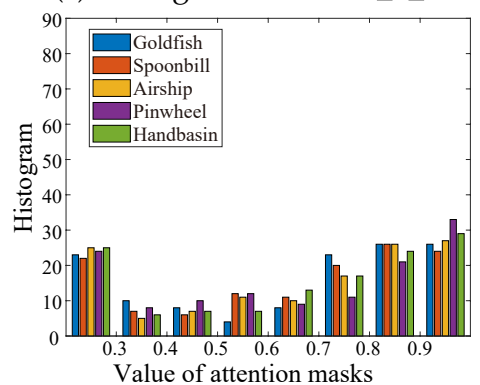

(f) Histogram of GPCA_3_1

Fig. 8: The values of the attention masks in (a) the SENet and (d) the GPCA modules with the ResNet18 on the ImageNet$32 \times 32$ dataset. We also zoomed in channel 70-80 (dashed boxes in (a) and (d)) for better illustration in (b) and (e), respectively. The histograms of the values are illustrated in (c) for the SENet and in (f) for the GPCA, respectively. The modules in each subfigure is named as "attention name_stageID_blockID".

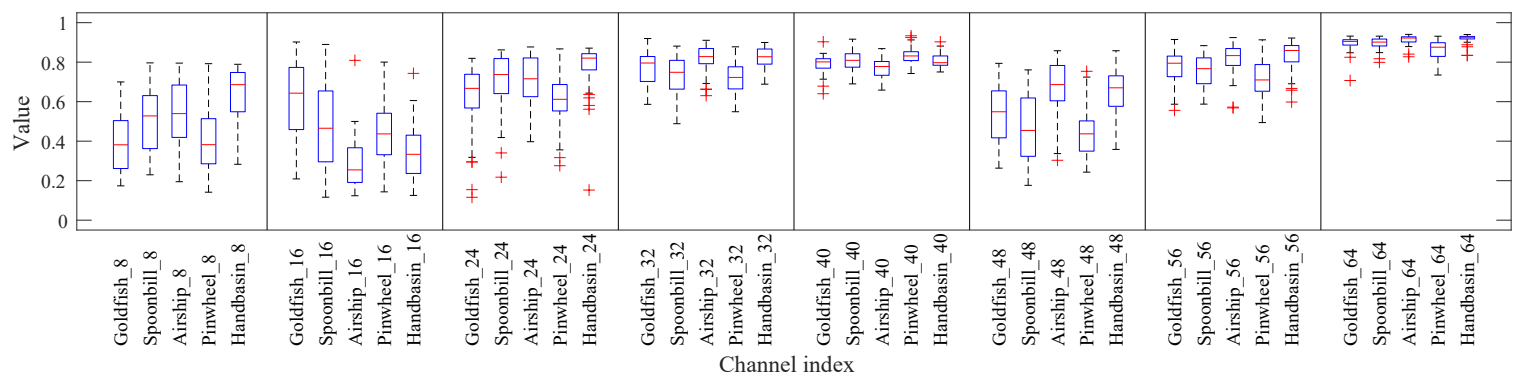

(a) GPCA_2_1

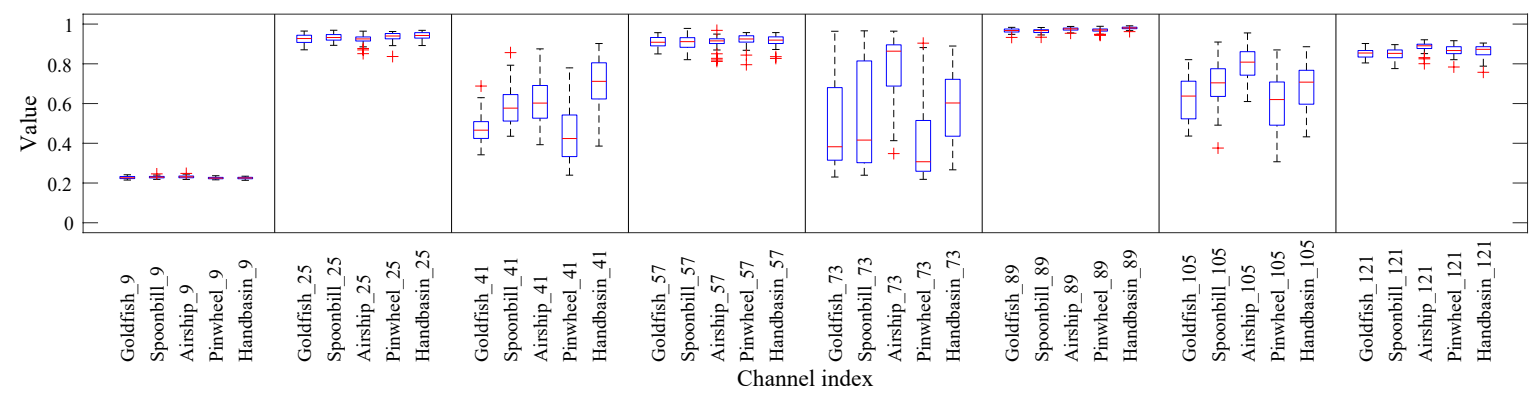

(b) GPCA_3_1

Fig. 9: Boxplots of the attention masks at different depths in the GPCA module with the ResNet1 8 on the ImageNet-32 332 dataset. The $x$-axis denotes the channel index. The modules in each subfigure are named as GPCA_stageID_blockID. GPCA_2_1 and GPCA_3_1 are selected as examples. For better illustration, we selected parts of the channels and draw the corresponding boxplots.

We conduct a group of experiments with standard negative log-likelihood (NLL) metric [78] (the smaller the better). According to Table 11, the GPCA module obtains the smallest NLL values on all the three base models, compared with the SENet and the SRP. This indicates that the GPCA can learn the channel correlations better than the referred methods. It clearly explains why the GPCA essentially performs better than the full connection-based method (e.g., the SENet) and the convolution- and pooling-based method (e.g., the SRP).
TABLE 11: Analysis of GPCA module by comparing the NLLs on the CUB-200-2011 dataset. The means and standard deviations of three rounds of evaluations are listed. The best results for each base model are marked in bold.

\begin{tabular}{lccc}
\hline \multicolumn{1}{c}{ Method } & \multicolumn{1}{c}{ VGG16 } & \multicolumn{1}{c}{ MobileNet } & ResNet50 \\
\hline SENet (2018) & $4.6127 \pm 0.4220$ & $3.6650 \pm 0.0899$ & $3.6200 \pm 0.1052$ \\
SRP (2019) & $5.0990 \pm 0.0016$ & $5.0961 \pm 0.0052$ & $5.0814 \pm 0.0179$ \\
GPCA (ours) & $\mathbf{3 . 5 9 6 9} \pm \mathbf{0 . 2 2 1 7}$ & $\mathbf{3 . 5 5 3 7} \pm \mathbf{0 . 0 5 5 5}$ & $\mathbf{3 . 4 7 2 9} \pm \mathbf{0 . 2 3 9 0}$ \\
\hline
\end{tabular}


TABLE 12: Comparisons of parameter numbers (\#Params.), computational complexities, and training and inference times per epoch (second/epoch). $C$ is number of channels, $H$ and $W$ are height and width of feature maps, and $|\cdot|_{\text {ood }}$ indicates the nearest odd number. The training and inference times were evaluated in the ResNet50 on the Cifar-100 dataset with batch size as 256 using one NVIDIA GTX1080Ti.

\begin{tabular}{|c|c|c|c|c|}
\hline Model & \#Params. & Computational complexity & Training time & Inference time \\
\hline SENet (2018) & $\frac{2 \times C^{2}}{r}$ with $r=4$ & $O\left(C^{2}\right)$ & 26.41 & 1.49 \\
\hline NLNet (2018) & ${ }^{r} \quad 2 C^{2}$ & $O\left(C^{2}+H^{2} W^{2} C\right)$ & 44.38 & 4.52 \\
\hline BAM (2018) & $\frac{3 \times C^{2}+C}{r}+\frac{9 C^{2}}{r^{2}}$ with $r=4$ & $O\left(C^{2}\right)$ & 21.16 & 2.13 \\
\hline CBAM (2018) & $\frac{2 C^{2}}{r}+98$ with $r=4$ & $O\left(C^{2}\right)$ & 36.63 & 3.64 \\
\hline ECA $(2020)$ & $\left|\frac{\log _{2} C}{\gamma}+\frac{b}{\gamma}\right| \quad$ with $\gamma=2, b=1$ & $O(C \ln C)$ & 22.30 & 2.43 \\
\hline GCT (2020) & $3 \times C$ & $O\left(C^{2}\right)$ & 25.53 & 2.59 \\
\hline GPCA (ours) & 4 & $O\left(C^{3}\right)$ & 53.19 & 5.22 \\
\hline
\end{tabular}

TABLE 13: Two simplified versions of the proposed GPCA module by local Gaussian process (Local) and multihead attention (MHA). We compare their performance on three datasets, including Cifar-10/-100 and ImageNet. Moreover, number of parameters, computational complexity, and training and inference time per epoch (second/epoch) are also compared. $h$ is head number in the MHA.

\begin{tabular}{|c|c|c|c|c|c|c|c|c|}
\hline \multirow{2}{*}{ Method } & \multirow{2}{*}{ Cifar-10 } & \multirow{2}{*}{ Cifar-100 } & \multicolumn{2}{|c|}{ ImageNet } & \multirow{2}{*}{ \#Params. } & \multirow{2}{*}{$\begin{array}{l}\text { Computational } \\
\text { complexity }\end{array}$} & \multirow{2}{*}{$\begin{array}{c}\text { Training } \\
\text { time }\end{array}$} & \multirow{2}{*}{$\begin{array}{l}\text { Inference } \\
\text { time }\end{array}$} \\
\hline & & & Top-1 & Top-5 & & & & \\
\hline GPCA-Local & $93.73 \pm 0.21$ & $72.02 \pm 0.15$ & $76.36 \pm 0.13$ & $93.41 \pm 0.17$ & 4 & $O\left((\ln C)^{3}\right)$ & 24.15 & 2.39 \\
\hline GPCA-MHA & $94.11 \pm 0.09$ & $72.37 \pm 0.12$ & $76.42 \pm 0.16$ & $93.38 \pm 0.26$ & 4 & $O\left(\left(\frac{C}{h}\right)^{3}\right)$ & 33.95 & 3.30 \\
\hline GPCA & $94.93 \pm 0.01$ & $73.97 \pm 0.02$ & $77.57 \pm 0.07$ & $93.97 \pm 0.06$ & 4 & $O\left(C^{3}\right)$ & 53.19 & 5.22 \\
\hline
\end{tabular}

\subsection{Comparisons of Parameter Numbers and Computa- tional Complexities}

We compare the numbers of parameters and computational complexity of the proposed GPCA module and the referred attention modules, respectively, as shown in Table 12 . The proposed GPCA module only introduces the four parameters in $\Theta$, which is much less than the other attention modules, and the computational complexity of it is $O\left(C^{3}\right)$, which is a little higher than them. We further compare the training and the inference times per epoch (in second/epoch). It can be observed that the training and inference times of GPCA are not increased too much and have similar values to those of the NLNet.

\subsection{Two Simplified Versions of GPCA}

In order to evaluate the trade-off between the performance and the computational complexity, we propose two simplified versions of the GPCA with lower computational complexities. The first version introduces the local GP as the prior, in which the GP prior is conducted in a near neighbourhood of the channels, namely GPCA-Local. In the GPCA-Local, $\left|\frac{\log _{2} C}{\gamma}+\frac{b}{\gamma}\right|_{\text {ood }}$ channels with $\gamma=2, b=1$ were selected for one channel as the local neighbourhood, similar to the ECA module. Another version introduces the multihead attention (MHA)-like framework, assigning channels into different groups and conducting the GPCA inside each group (denoted as GPCA-MHA from hereby). In the GPCA-MHA, we assigned 16 consecutive channels into one group for each block and obtained $\frac{C}{16}$ groups in total. The comparisons between these two versions and the full GPCA are shown in Table 13. Although these two simplified versions can significantly reduce the computational complexities and the time cost, the performance in the image classification task is also decreased. This is due to a trade-off between the performance and the computational complexity.

\section{Conclusions}

In this paper, we proposed a Gaussian process embedded channel attention (GPCA) module to learn the correlations among the channels in CNNs via a probabilistic way. In the proposed GPCA, the output channel attention masks were assumed as beta distributed variables. We adapted a Sigmoid-Gaussian approximation, in which the Gaussian distributed variables were transferred and bounded into the interval of $[0,1]$ by a Sigmoid function. The Gaussian process was utilized to model the correlations among different channels and applied as a prior of the channel attention masks. In this case, the proposed GPCA module can be intuitively and reasonably clarified in a probabilistic way. Although the beta distribution has difficulty to be integrated into the end-to-end training of the $\mathrm{CNNs}$, we utilized an appropriate approximation of the beta distribution to derive an analytically tractable solution to facilitate the calculations.

Furthermore, experimental results show that the proposed GPCA can statistically significantly improve the classification accuracies with three widely used base models on five benchmark image classification datasets, compared with eight referred attention modules. In addition, statistically significant improvements have also been obtained in WSOL, object detection, and semantic segmentation tasks, compared with recently proposed state-of-the-art methods.

\section{ACKNOWLEDGMENTS}

This work was supported in part by the National Key R\&D Program of China under Grant 2019YFF0303300, Subject II No. 2019YFF0303302, and 2020AAA0105200, in part by National Natural Science Foundation of China (NSFC) under Grant 61922015, 61773071, U19B2036, in part by Beijing Natural Science Foundation Project under Grant Z200002, and in part by BUPT Excellent Ph.D. Students Foundation No. CX2020105. 


\section{REFERENCES}

[1] K. Simonyan and A. Zisserman, "Very deep convolutional networks for large-scale image recognition," in International Conference on Learning Representations, 2015.

[2] K. He, X. Zhang, S. Ren, and J. Sun, "Deep residual learning for image recognition," in Computer Vision and Pattern Recognition, 2016, pp. 770-778.

[3] G. Huang, Z. Liu, L. V. Der Maaten, and K. Q. Weinberger, "Densely connected convolutional networks," in Computer Vision and Pattern Recognition, 2017, pp. 2261-2269.

[4] Z. Peng, Z. Li, J. Zhang, Y. Li, G.-J. Qi, and J. Tang, "Fewshot image recognition with knowledge transfer," in International Conference on Computer Vision (ICCV), 2019, pp. 441-449.

[5] W. Liu, D. Anguelov, D. Erhan, C. Szegedy, S. E. Reed, C. Fu, and A. C. Berg, "SSD: Single shot multibox detector," in European Conference on Computer Vision, 2016, pp. 21-37.

[6] L. Chen, G. Papandreou, I. Kokkinos, K. Murphy, and A. L. Yuille, "DeepLab: Semantic image segmentation with deep convolutional nets, atrous convolution, and fully connected CRFs," IEEE Transactions on Pattern Analysis and Machine Intelligence, vol. 40, no. 4, pp. 834-848, 2018.

[7] Y. Yuan, X. Chen, and J. Wang, "Object-contextual representations for semantic segmentation," arXiv preprint arXiv:1909.11065, 2019.

[8] Z. Li, J. Tang, and T. Mei, "Deep collaborative embedding for social image understanding," IEEE Transactions on Pattern Analysis and Machine Intelligence, vol. 41, no. 9, pp. 2070-2083, 2019.

[9] G. E. Hinton, N. Srivastava, A. Krizhevsky, I. Sutskever, and R. Salakhutdinov, "Improving neural networks by preventing coadaptation of feature detectors," arXiv, 2012.

[10] A. Vaswani, N. Shazeer, N. Parmar, J. Uszko-reit, L. Jones, A. N. Gomez, Ł. Kaiser, and I. Polosukhin, "Attention is all you need," in Advances in Neural Information Processing Systems, 2017, pp. 59986008.

[11] J. Hu, L. Q. Shen, and G. Sun, "Squeeze-and-excitation networks," in Computer Vision and Pattern Recognition, 2018, pp. 7132-7141.

[12] M. Jaderberg, K. Simonyan, A. Zisserman, and K. Kavukcuoglu, "Spatial transformer networks," in Neural Information Processing Systems, 2015, pp. 2017-2025.

[13] S. Woo, J. Park, J.-Y. Lee, and I. S. Kweon, "CBAM: Convolutional block attention module," in European Conference on Computer Vision, 2018, pp. 3-19.

[14] J. Hu, L. Shen, S. Albanie, G. Sun, and A. Vedaldi, "Gather-Excite: Exploiting feature context in convolutional neural networks," in Neural Information Processing Systems, 2018, pp. 9401-9411.

[15] M. Sun, Y. Yuan, F. Zhou, and E. Ding, "Multi-attention multiclass constraint for fine-grained image recognition," in European Conference on Computer Vision, 2018, pp. 834-850.

[16] J. Park, S. Woo, J. Lee, and I. S. Kweon, "BAM: Bottleneck attention module.," in British Machine Vision Conference, 2018, p. 147.

[17] Z. Huang, S. Liang, M. Liang, and H. Yang, "DIANet: Dense-andimplicit attention network.," arXiv, 2019.

[18] Y. Yang, X. Wang, Q. Zhao, and T. Sui, "Two-level attentions and grouping attention convolutional network for fine-grained image classification," Applied Sciences, vol. 9, no. 9, pp. 1939, 2019.

[19] M. Luo, G. Wen, Y. Hu, D. Dai, and Y. Xu, "Stochastic region pooling: Make attention more expressive.," arXiv, 2019.

[20] P. R. Lopez, D. V. Dorta, G. C. Preixens, J. M. G. Sitjes, F. X. R. Marva, and J. Gonzalez, "Pay attention to the activations: a modular attention mechanism for fine-grained image recognition," IEEE Transactions on Multimedia, 2019.

[21] C. M. Bishop, Pattern recognition and machine learning, Springer Science+Business Media LLC., 2006.

[22] C. E. Rasmussen and C. K. I. Williams, Gaussian processes for machine learning, The MIT Press, 2006.

[23] B. Chen and W. Deng, "Hybrid-attention based decoupled metric learning for zero-shot image retrieval," in Computer Vision and Pattern Recognition, 2019, pp. 2745-2754.

[24] P. Fang, J. Zhou, S. Roy, L. Petersson, and M. Harandi, "Bilinear attention networks for person retrieval," in International Conference on Computer Vision, 2019, pp. 8029-8038.

[25] T. Zhao and $\mathrm{X}$. Wu, "Pyramid feature attention network for saliency detection," in Computer Vision and Pattern Recognition, 2019, pp. 3080-3089.

[26] S. Chen, X. Tan, B. Wang, and X. Hu, "Reverse attention for salient object detection," in European Conference on Computer Vision, 2018, pp. 236-252.
[27] D. Linsley, D. Shiebler, S. Eberhardt, and T. Serre, "Global-andlocal attention networks for visual recognition," arXiv, 2018.

[28] B. Chen, W. Deng, and J. Hu, "Mixed high-order attention network for person re-identification," in International Conference on Computer Vision, 2019, pp. 371-381.

[29] G. Chen, C. Lin, L. Ren, J. Lu, and J. Zhou, "Self-critical attention learning for person re-identification," in International Conference on Computer Vision, 2019, pp. 9636-9645.

[30] Y. Yan, B. Ni, J. Liu, and X. Yang, "Multi-level attention model for person re-identification," Pattern Recognition Letters, 2018.

[31] Z. Zhu, W. Wu, W. Zou, and J. Yan, "End-to-end flow correlation tracking with spatial-temporal attention," in Computer Vision and Pattern Recognition, 2018, pp. 548-557.

[32] A. Muqeet, T. B. Iqbal, and S. Bae, "Hybrid residual attention network for single image super resolution," arXiv, 2019.

[33] D. Jang and R. Park, "DenseNet with deep residual channelattention blocks for single image super resolution," in Computer Vision and Pattern Recognition, 2019.

[34] T. Dai, J. Cai, Y. Zhang, S. Xia, and L. Zhang, "Second-order attention network for single image super-resolution," in Computer Vision and Pattern Recognition, 2019, pp. 11057-11066.

[35] J. Fu, J. Liu, H. Tian, Y. Li, Y. Bao, Z. Fang, and H. Lu, "Dual attention network for scene segmentation," in Computer Vision and Pattern Recognition, 2019, pp. 3141-3149.

[36] Z. Huang, X. Wang, L. Huang, C. Huang, Y. Wei, and W. Liu, "CCNet: Criss-cross attention for semantic segmentation," in International Conference on Computer Vision, 2019, pp. 603-612.

[37] X. Lu, W. Wang, C. Ma, J. Shen, L. Shao, and F. Porikli, "See more, know more: Unsupervised video object segmentation with co-attention siamese networks," in Computer Vision and Pattern Recognition, 2019, pp. 3618-3627.

[38] I. Bello, B. Zoph, Q. Le, A. Vaswani, and J. Shlens, “Attention augmented convolutional networks," in International Conference on Computer Vision, 2019, pp. 3285-3294.

[39] Y. Cao, J. Xu, S. Lin, F. Wei, and H. Hu, "GCNet: Non-local networks meet squeeze-excitation networks and beyond," in International Conference on Computer Vision Workshop, 2019, pp. 1971-1980

[40] Q. Wang, B. Wu, P. Zhu, P. Li, W. Zuo, and Q. Hu, "ECANet: Efficient channel attention for deep convolutional neural networks," in Computer Vision and Pattern Recognition, 2020.

[41] Z. Yang, L. Zhu, Y. Wu, and Y. Yang, "Gated channel transformation for visual recognition," in Computer Vision and Pattern Recognition, 2020.

[42] S. Hochreiter and J. Schmidhuber, "Long short-term memory," Neural Computation, vol. 9, no. 8, pp. 1735-1780, 1997.

[43] S. Sudhakaran, S. Escalera, and O. Lanz, "LSTA: Long short-term attention for egocentric action recognition," in Computer Vision and Pattern Recognition, 2019, pp. 9946-9955.

[44] X. Li, W. Wang, X. Hu, and J. Yang, "Selective kernel networks," in Computer Vision and Pattern Recognition, 2019.

[45] Y. Chen, X. Dai, M. Liu, D. Chen, L. Yuan, and Z. Liu, "Dynamic convolution: Attention over convolution kernels," in Computer Vision and Pattern Recognition, 2020.

[46] T. Lin, A. Roychowdhury, and S. Maji, "Bilinear CNN models for fine-grained visual recognition," in International Conference on Computer Vision, 2015, pp. 1449-1457.

[47] Z. Zheng, G. An, D. Wu, and Q. Ruan, "Global and local knowledge-aware attention network for action recognition," IEEE Transactions on Neural Networks and Learning Systems, pp. 1-14, 2020.

[48] L. Yang, Q. Song, Y. Wu, and M. Hu, "Attention inspiring receptive-fields network for learning invariant representations," IEEE Transactions on Neural Networks and Learning Systems, vol. 30, no. 6, pp. 1744-1755, 2019.

[49] X. Wang, R. Girshick, A. Gupta, and K. He, "Non-local neural networks," in Computer Vision and Pattern Recognition, June 2018 pp. 7794-7803.

[50] J. Choe and H. Shim, "Attention-based dropout layer for weakly supervised object localization," in Computer Vision and Pattern Recognition, 2019, pp. 2214-2223.

[51] J. Choe, S. Lee, and H. Shim, "Attention-based dropout layer for weakly supervised single object localization and semantic segmentation," IEEE Transactions on Pattern Analysis and Machine Intelligence, 2020

[52] H. Zhao, J. Jia, and V. Koltun, "Exploring self-attention for image recognition," in Computer Vision and Pattern Recognition, 2020. 
[53] J. Xie, Z. Ma, G. Zhang, J.-H. Xue, Z.-H. Tan, and J. Guo, "Soft dropout and its variational Bayes approximation," in IEEE International Workshop on Machine Learning for Signal Processing, 2019.

[54] C. J. Maddison, A. Mnih, and Y. W. Teh, "The concrete distribution: A continuous relaxation of discrete random variables," ArXiv, 2017.

[55] D. J. C. MacKay, "The evidence framework applied to classification networks," Neural Computation, vol. 4, no. 5, pp. 720-736, 1992.

[56] A. Krizhevsky, "Learning multiple layers of features from tiny images," techreport, CIFAR, 2009.

[57] O. Vinyals, C. Blundell, T. P. Lillicrap, K. Kavukcuoglu, and D. Wierstra, "Matching networks for one shot learning," arXiv, 2016.

[58] A. V. Den Oord, N. Kalchbrenner, and K. Kavukcuoglu, "Pixel recurrent neural networks," arXiv, 2016.

[59] O. Russakovsky, J. Deng, H. Su, J. Krause, S. Satheesh, S. Ma, Z. Huang, A. Karpathy, A. Khosla, M. S. Bernstein, A. C. Berg, and L. Feifei, "ImageNet large scale visual recognition challenge," International Journal of Computer Vision, vol. 115, no. 3, pp. 211-252, 2015.

[60] R. R. Selvaraju, M. Cogswell, A. Das, R. Vedantam, D. Parikh, and D. Batra, "Grad-CAM: Visual explanations from deep networks via gradient-based localization," in International Conference on Computer Vision, 2017, pp. 618-626.

[61] B. Zhou, A. Khosla, A. Lapedriza, A. Oliva, and A. Torralba, "Learning deep features for discriminative localization," in Computer Vision and Pattern Recognition, 2016, pp. 2921-2929.

[62] X. Zhang, Y. Wei, J. Feng, Y. Yang, and T. Huang, "Adversarial complementary learning for weakly supervised pbject localization," in Computer Vision and Pattern Recognition, 2018, pp. 13251334.

[63] K. K. Singh and Y. J. Lee, "Hide-and-seek: Forcing a network to be meticulous for weakly-supervised object and action localization," in International Conference on Computer Vision, 2017, pp. 3544-3553.

[64] X. Zhang, Y. Wei, G. Kang, Y. Yang, and T. S. Huang, "Selfproduced guidance for weakly-supervised object localization," in European Conference on Computer Vision, 2018, pp. 610-625.

[65] H. Xue, C. Liu, F. Wan, J. Jiao, X. Ji, and Q. Ye, "DANet: Divergent activation for weakly supervised object localization," in International Conference on Computer Vision, 2019, pp. 6588-6597.

[66] J. Yin, S. Zhang, D. Chang, Z. Ma, and J. Guo, "Dual-attention guided dropblock module for weakly supervised object localization," in International Conference on Pattern Recognition, 2020.

[67] C. Wah, S. Branson, P. Welinder, P. Perona, and S. Belongie, "The caltech-ucsd birds-200-2011dataset," http://www.vision.caltech. edu/visipedia/cub-200-2011.html. 2011.

[68] A. G. Howard, M. Zhu, B. Chen, D. Kalenichenko, W. Wang, T. Weyand, M. Andreetto, and H. Adam, "MobileNets: Efficient convolutional neural networks for mobile vision applications," arXiv, 2017.

[69] “The PASCAL visual object classes challenge (VOC2007)," http:// www.pascal-network.org/challenges/VOC/voc2007/index.html

[70] T. Lin, M. Maire, S. J. Belongie, J. Hays, P. Perona, D. Ramanan, P. Dollar, and C. L. Zitnick, "Microsoft COCO: Common objects in context," in European Conference on Computer Vision, 2014, pp. 740-755.

[71] Z. Tian, C. Shen, H. Chen, and T. He, "FCOS: Fully convolutional one-stage object detection," in International Conference on Computer Vision, 2019.

[72] S. Zhang, C. Chi, Y. Yao, Z. Lei, and S. Z. Li, "Bridging the gap between anchor-based and anchor-free detection via adaptive training sample selection," in Computer Vision and Pattern Recognition, June 2020.

[73] T. Lin, P. Dollár, R. Girshick, K. He, B. Hariharan, and S. Belongie, "Feature pyramid networks for object detection," in Computer Vision and Pattern Recognition, 2017, pp. 936-944.

[74] K. Chen, J. Wang, J. Pang, Y. Cao, Y. Xiong, X. Li, S. Sun, W. Feng, Z. Liu, J. Xu, Z. Zhang, D. Cheng, C. Zhu, T. Cheng, Q. Zhao, B. Li, X. Lu, R. Zhu, Y. Wu, J. Dai, J. Wang, J. Shi, W. Ouyang, C.-C. Loy, and D. Lin, "MMDetection: Open mmlab detection toolbox and benchmark," arXiv preprint arXiv:1906.07155, 2019.

[75] MMSegmentation Contributors, "MMSegmentation: Openmmlab semantic segmentation toolbox and benchmark," https://github. com/open-mmlab/mmsegmentation, 2020.

[76] Ma. Cordts, M. Omran, S. Ramos, T. Rehfeld, M. Enzweiler, R. Benenson, U. Franke, S. Roth, and B. Schiele, "The cityscapes dataset for semantic urban scene understanding," in Computer Vision and Pattern Recognition, 2016.

[77] K. Sun, B. Xiao, D. Liu, and J. Wang, "Deep high-resolution representation learning for human pose estimation," in Computer Vision and Pattern Recognition, 2019, pp. 5693-5703.

[78] W. J. Maddox, P. Izmailov, T. Garipov, D. Vetrov, and A. G. Wilson, "A simple baseline for Bayesian uncertainty in deep learning," in Neural Information Processing Systems, 2019, pp. 13153-13164. 